\title{
Postnatal Hematopoiesis and Gut Microbiota in NOD Mice Deviate from C57BL/6 Mice
}

\author{
Dina Silke Malling Damlund, ${ }^{1}$ Stine Broeng Metzdorff, ${ }^{1}$ Jane Preuss Hasselby, ${ }^{2}$ \\ Maria Wiese, ${ }^{3}$ Mia Lundsager, ${ }^{1}$ Dennis Sandris Nielsen, ${ }^{3}$ Karsten Stig Buschard, ${ }^{4}$ \\ Axel Kornerup Hansen, ${ }^{1}$ and Hanne Frøkiær ${ }^{1}$
}

\author{
${ }^{1}$ Department of Veterinary Disease Biology, Faculty of Health and Medical Sciences, University of Copenhagen, \\ 1870 Frederiksberg C, Denmark \\ ${ }^{2}$ Department of Pathology, Rigshospitalet, 2100 Copenhagen, Denmark \\ ${ }^{3}$ Department of Food Science, University of Copenhagen, 1870 Frederiksberg C, Denmark \\ ${ }^{4}$ Bartholin Institute, Rigshospitalet, 2100 Copenhagen, Denmark
}

Correspondence should be addressed to Dina Silke Malling Damlund; msilke@sund.ku.dk

Received 20 May 2015; Revised 9 July 2015; Accepted 21 July 2015

Academic Editor: Urs Christen

Copyright (C) 2016 Dina Silke Malling Damlund et al. This is an open access article distributed under the Creative Commons Attribution License, which permits unrestricted use, distribution, and reproduction in any medium, provided the original work is properly cited.

\begin{abstract}
Neonatal studies in different mouse strains reveal that early life colonization affects the development of adaptive immunity in mice. The nonobese diabetic (NOD) mouse spontaneously develops autoimmune diabetes, but neonatal studies of NOD mice are lacking. We hypothesized that NOD mice deviate from another much used mouse strain, C57BL/6, with respect to postnatal microbiota and/or hematopoiesis and compared this in newborn mice of dams housed under the same conditions. A distinct bacteria profile rich in staphylococci was found at postnatal days (PND) 1-4 in NOD mice. Furthermore, a distinct splenic cell profile high in a granulocytic phenotype was evident in the neonatal NOD mice whereas neonatal C57BL/6 mice showed a profile rich in monocytes. Neonatal expression of Reg3g and Muc2 in the gut was deviating in NOD mice and coincided with fewer bacteria attaching to the Mucosal surface in NOD compared to C57BL/6 mice.
\end{abstract}

\section{Introduction}

The nonobese diabetic (NOD) mouse spontaneously develops autoimmune diabetes and provides a suitable rodent model for research in type 1 diabetes pathogenesis $[1,2]$. Host genetics, gut microbiota, and diet-associated factors are central in the development of diabetes in NOD mice [37]. However, the mechanisms behind the microbiota-host interaction and the specific genetic deviations leading to the disease pathology of type 1 diabetes are poorly understood.

The development of autoimmune diabetes in NOD mice is a result of polygenic interactions. The etiology is complex $[5,8]$, with genetics playing an important role, but also other factors influence the disease pathology [9]. The incidence of diabetes in NOD mice has been correlated with hygiene and microbiota in the colony and housing facility $[4,10]$ and gut microbiota (GM) dysbiosis have been linked to type 1 diabetes development $[11,12]$. Factors determining GM composition are complex. Type of delivery and environmental factors have been implicated to play important roles in the establishment of GM. Children delivered by caesarean section may display an increased risk to develop autoimmune diabetes [13] indicating that early life colonization influences the development of type 1 diabetes.

Postnatal development of the immune system is heavily influenced by GM [14]. Germ-free mice have an underdeveloped mucosal epithelium with smaller Peyer's patches, fewer isolated lymphoid follicles, and reduced production of antimicrobial peptides [15]. GM composition is influenced by antimicrobial peptides [16] and one function of the mucus 
layer is to retain defensins and antimicrobial proteins, such as the antimicrobial C-type lectin Reg3g, in close proximity to the epithelial surface [17]. Reg3g is normally expressed from weaning and restricts the number of surface-associated bacteria [16], mainly targeting mucosa-associated Gram-positive bacteria [18]. In inflammatory bowel disease expression of the secreted mucus protein Muc2 is downregulated [19] while Reg3g expression is increased [20] indicating that Reg3g expression increases when the mucus layer is disassembled.

It has been suggested that genetic defects involved in the development of type 1 diabetes in NOD mice are linked to differentiation and proliferation of hematopoietic stem cells (HSCs) [21, 22]. Notably, bone marrow transplantation from young $\mathrm{BALB} / \mathrm{c}$ mice to NOD mice prevents and reverses the development of autoimmune diabetes [21]. Langmuir et al. [23] demonstrated that expression of the hematopoietic differentiation antigen Ly6C, expressed on monocytes, neutrophils, and T cells $[24,25]$, is severely impaired in NOD mice. An interruption in the flanking region of the Ly6C gene upstream of the transcription initiation site is present in the NOD mice genome [26], which appears to hamper the transcription. The Ly6C gene is not completely knocked out in NOD mice, as some Ly6C expression is still detectable. Stimulation by cytokines does not restore the expression of the gene [26].

Knowledge regarding deviations in the development and differentiation of HSCs and defects in myelopoiesis as well as gut colonisation and gene expression here might aid to understand autoimmunity in NOD mice. We therefore compared development and activation of the myeloid cell lineage and GM composition in NOD and C57BL/6 mice focusing on the very first days after birth and found significant differences between the two strains, as regards both neonatal GM and hematopoiesis.

We speculate that NOD mice in the neonatal period have an altered GM, which in turn may influence the development of the genetically deviating immune system.

\section{Experimental Procedures}

2.1. Animals and Tissue. NOD/BomTac and C57BL/6 mice were purchased from Taconic (Hudson, NY, USA, and Denmark, resp.) and housed in a barrier protected rodent facility (Faculty of Health and Medical Science, University of Copenhagen, Frederiksberg, Denmark) under standard conditions in open cages without filter and with access to water and food (Altromin 1324, Lage, Germany) ad libitum. The two strains were mated separately and postnatal day 1 (PND 1) was defined as the day pups were born. 16-18 pubs from each strain were euthanized at PND 1, 2, 4, 7, 21, 28, and 70 by decapitation (PND 1-7) and cervical dislocation (PND 21-70). 6-8 spleens from each strain were dissected, kept on ice, and prepared for flow cytometry. 4-6 spleens, liver, and intestine samples were transferred to RNAlater (Ambion) for RNA and DNA extraction and further 2-3 spleens and livers were collected for histopathology examinations.

The project was licensed by the National Committee for Animal Experimentation under the Danish Ministry of Food, Fisheries and Agriculture (http://www.indberetning
TABLE 1: Gene expression assays used for qPCR.

\begin{tabular}{lcc}
\hline Gene & Gene name & Assay ID \\
\hline Actb & Actin, beta & Mn00607939_s1 \\
Elane & Elastase, neutrophil expressed & Mm01168928_g1 \\
Arg1 & Arginase & Mm00475988_m1 \\
Hp & Haptoglobin & Mn00516884_m1 \\
Camp & Cathelicidin antimicrobial peptide & Mm00438285_m1 \\
Reg3g & Regenerating islet-derived 3 gamma & Mm00441127_m1 \\
Muc2 & Mucin 2 & Mm01276696_m1 \\
\hline
\end{tabular}

.dyreforsoegstilsynet.dk/) operating according to the EU directive 2010/63/EU (license number 2012-15-2934-00256$\mathrm{C} 1)$.

2.2. Splenic Cell Suspensions. Preparation of single cell suspensions was carried out by mechanical disruption of the spleen. Splenocytes were washed in ice cold sterile RPMI 1640 (Lonza), centrifuged for $10 \mathrm{~min}\left(200 \mathrm{~g}, 4^{\circ} \mathrm{C}\right)$. Erythrocytes were lysed by resuspension in $4 \mathrm{~mL} 0.2 \%$ sterile filtrated saline for $30 \mathrm{sec}$., following addition of $4 \mathrm{~mL} 1.6 \%$ saline. The procedure was repeated until the pellet was visibly free of erythrocytes. Cells were washed in RPMI, before viable cell count.

2.3. Analysis of Cell Population. Splenic single cell suspensions $\left(1 \times 10^{5}-1 \times 10^{6}\right.$ cells/well $)$ were incubated in the dark for $10 \mathrm{~min}$ with $3 \mu \mathrm{g} / \mathrm{mL}$ anti-CD16/32 (BD Biosciences, Franklin Lakes, NJ, USA). Subsequently the following antibodies were added: anti-CD11b PE-cy7 clone M1/70, anti-Ly6C PE clone AL-21, and Ly6G APC clone 1A8 (eBiosciences, San Diego, CA, USA). Cells were analysed immediately on a FACScanto flow cytometer (BD Biosciences). Data analysis and layouts of FACS data were performed using Flowjo V 10.6 (Tree Star, Ashland, OR, USA).

2.4. RNA Isolation, cDNA Synthesis, and Gene Expression Analysis by qPCR. RNA extraction from samples stored in RNAlater and subsequently cDNA synthesis were performed as previously described [27].

Gene expression of Reg3g, Camp, Arg1, Elane, Hp, Muc2, and $A c t b$ was analysed as previously described [27] in samples from spleen, liver, and intestine using TaqMan Gene Expression Assays (Life Technology, Table 1). Fold changes in gene expression were calculated as $2^{-\Delta \Delta \mathrm{Ct}}$ using the $\Delta \Delta \mathrm{Ct}$ method [28]. Briefly the expression of each sample was normalized to the expression of the reference $(A c t b)$ gene: $(\Delta \mathrm{Ct}=$ $\mathrm{Ct}$ (target) - Ct(reference) $)$, and the relative gene expression was calculated as $(\Delta \Delta \mathrm{Ct}=\Delta \mathrm{Ct}($ target $)-\Delta \mathrm{Ct}$ (control) $)$, where $\Delta \mathrm{Ct}$ (control) is the average $\Delta \mathrm{Ct}$ from NOD PND 1 samples.

2.5. MiSeq-Based 16S rRNA Gene Amplicon High Throughput Sequencing. 31 mouse GM profiles were determined using tag-encoded 16S rRNA gene amplicon MiSeq (Illumina) based high throughput sequencing. DNA extraction and 16S rRNA gene library preparation were performed as previously described [29]. 
The raw dataset was trimmed using the "trim sequences" tool in CLC Genomic Workbench (CLC bio, Aarhus, Denmark). The fasta file format was made compatible with the Quantitative Insight Into Microbial Ecology (QIIME) open source software package (1.8.0) with an in-house Matlab script. Purging the dataset from chimeric reads and OTU clustering was conducted using UPARSE-. TU [30]. The relative distribution of the genera registered was calculated for unified and summarized in the genus level OTU tables. Alpha and beta-diversity measures, including analysis of group differences based on uniFrac distance metrics and ANOSIM analysis, were determined and analysed as previously described [29].

2.6. Generation and Harvest of Murine Bone Marrow-Derived Cells. Bone marrow cells were obtained from femur and tibia from adult NOD and C57BL/6 mice as previously described [31]. Cell suspensions were diluted to a concentration of 3 $\times 10^{5}$ cells $/ \mathrm{mL}$ containing GM-CSF and incubated at $37^{\circ}$ in a $5 \% \mathrm{CO}_{2}$-incubator (day 0). On day 3 the cultured cells were stimulated with Escherichia coli Nissle 1917 (MOI 0.1), Lactobacillus acidophilus NCFM (MOI 0.1), or a mix of both (MOI 0.05 each). The cells were harvested at day 6, stained and analysed by flow cytometry.

\subsection{Hematoxylin and Eosin (HE) Staining and Gram Staining.} Spleens, liver, and intestine were formalin fixed and paraffin embedded, cut into $4 \mu \mathrm{m}$ thin sections, and mounted on superfrost plus slides and HE stained. The slides were evaluated by blinded visual inspection including an experienced pathologist. The degree of granulocytic infiltration was scored as 0 (no infiltration), 1 (mild focal infiltration), 2 (moderate focal infiltration), or 3 (severe infiltration). The proportion of hematopoietic tissue was scored using similar criteria.

Liver and spleen tissue were cut into $4 \mu \mathrm{m}$ thin sections and Gram-stained as described by Bancroft et al. [32].

\subsection{Tissue Preparation and Projection Tomography (OPT)} Scanning. To visualize the intestinal anatomy at PND 1 in NOD and C57BL/6 mice (two mice from each strain) the intestine was dissected and processed for optical projection tomography (OPT) as earlier described [33]. Each sample was scanned using the Biotonics 3001 OPT scanner (Bioptonics, Edinburgh, UK) at $425 \mathrm{~nm}$ with a rotation degree of $45^{\circ}$ yielding 800 images with a resolution of $1024 \times 1024$ pixels. All samples were scanned at the same exposure time and same zoom factor. Scans were reconstructed with the NRecon V. 1.6.9.3 (Skyscan) software and digital sections illustrated in gray-scale demonstrating autofluorescence anatomy data were prepared with the Bioptonic Viewer Software V.2.0 (Bioptonics).

2.9. Statistical Analysis. For all statistical analysis, a twoway ANOVA test with Bonferroni post test was performed by Graph Pad Prism version 5.02 (Graphpad Software, San Diego, CA). In gene expression experiments, statistical analysis was performed on $\Delta \mathrm{Ct}$ values as these are assumed normally distributed as opposed to the fold change values.
Statistical significance was accepted at ${ }^{*} p<0.05,{ }^{* *} p<0.01$, and ${ }^{* * *} p<0.001$.

\section{Results}

3.1. Newborn NOD Mice Deviate in the $L y 6 G^{+} / L y 6 C^{+}$Splenocyte Population. To test our hypothesis that neonatal NOD mice are altered in their development of the immune system, we stained splenocytes from 1-7-day-old NOD and C57BL/6 mice with the CD11b, Ly6G, and Ly6C specific antibodies. Splenocytes from the two strains had significantly different proportions of $\mathrm{CD}_{11 b}{ }^{+}$cells at PND 1 (Figure 1(a)). Furthermore, they exhibited highly distinct expression profiles of the two myeloid lineage markers (Figures 1(b)-1(d)). Splenocytes from NOD mice held a high and increasing proportion of $\mathrm{CD}_{11 b^{+}} \mathrm{Ly}_{6 \mathrm{G}}{ }^{+} \mathrm{Ly}_{6} \mathrm{C}^{-}$cells from PND 1-7 (Figure 1(b)), a minor proportion of $\mathrm{CD} 1 \mathrm{~b}^{+} \mathrm{Ly} 6 \mathrm{G}^{-} \mathrm{Ly} 6 \mathrm{C}^{+}$ cells (Figure $1(\mathrm{c})$ ), and very little $\mathrm{CD}_{11 b^{+}} \mathrm{Ly} 6 \mathrm{G}^{+} \mathrm{Ly} 6 \mathrm{C}^{+}$cells (Figure 1(d)). In contrast, splenocytes from C57BL/6 mice

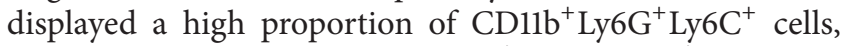

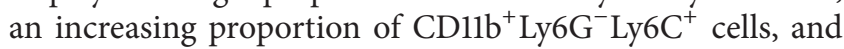
almost no CD11b ${ }^{+}$Ly6G ${ }^{+}$Ly6C $C^{-}$cells (Figures 1(b)-1(d)).

3.2. Livers of Newborn NOD Mice Are Rich in Cells with a Granulocytic Phenotype. The vast difference in the myeloid differentiation patterns in spleens from newborn NOD and C57BL/6 mice prompted us to inspect the hematopoietic tissue in the livers of the newborn mice as well as characterizing the phenotype of the cells present. Sections of haematoxylin and eosin (HE) stained livers were visually examined with regard to the number, type, and localization of myeloid derived differentiating cells (Figure 2(a)). No difference in the amount of hematopoietic tissue was observed (Figure 2(b)) and no difference was observed in the rate of differentiation (Figure 2(c)). Assessment of the number of differentiated cells in the liver sections demonstrated that, compared to C57BL/6 mice, NOD mice had a significant higher level of cells with a polylobed or ringed nuclei at PND 1,2, and 4 in the liver (Figure 2(c)). These cells were present in the liver as far as to PND 70 in NOD mice, though in much lower numbers than seen in the newborn mice.

Interestingly, in NOD mice the granulocytic phenotype of cells accumulated around the hepatic portal veins at PND 1 (Figure 3(a)), while in the C57BL/6 mice these cells were fewer and scattered in the tissue of the liver, with a gradient towards the blood vessels (Figure 3(b)). While the two mouse strains had similar numbers of hematopoietic stem cells in the livers at PND 1, they differed greatly in the number and in the localization of the differentiating granulocytic cells.

3.3. More Immature Granulocytes in the Spleen of NOD Mice Compared to C57BL/6 Mice. Visual examination of neonatal liver and spleen revealed a large proportion of granulocytic cells in different differentiation stages in NOD mice compared to C57BL/6 mice. To further assess their differentiation we measured the expression of Elane (neutrophil elastase), Arg1 (arginase 1), and Hp (haptoglobin), all genes expressed in various differentiation steps in granulocytes [34-36]. 


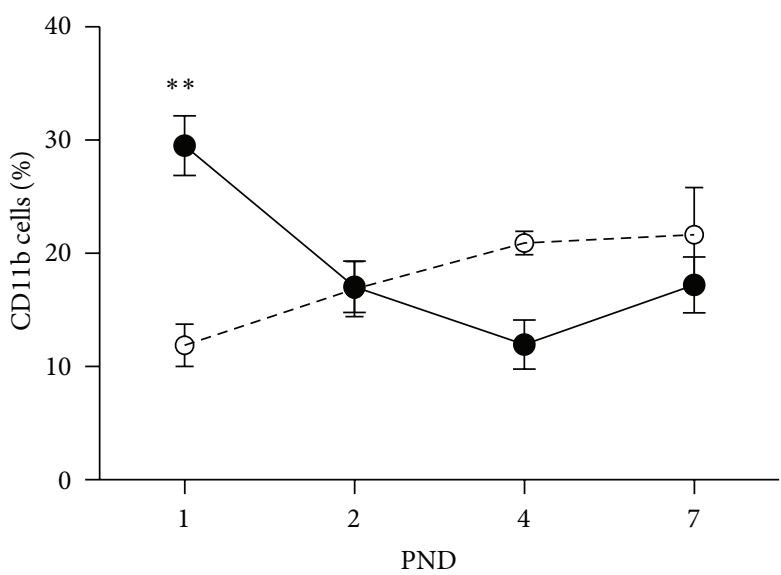

NOD

$-\Theta-\mathrm{C} 57 \mathrm{BL} / 6$

(a)

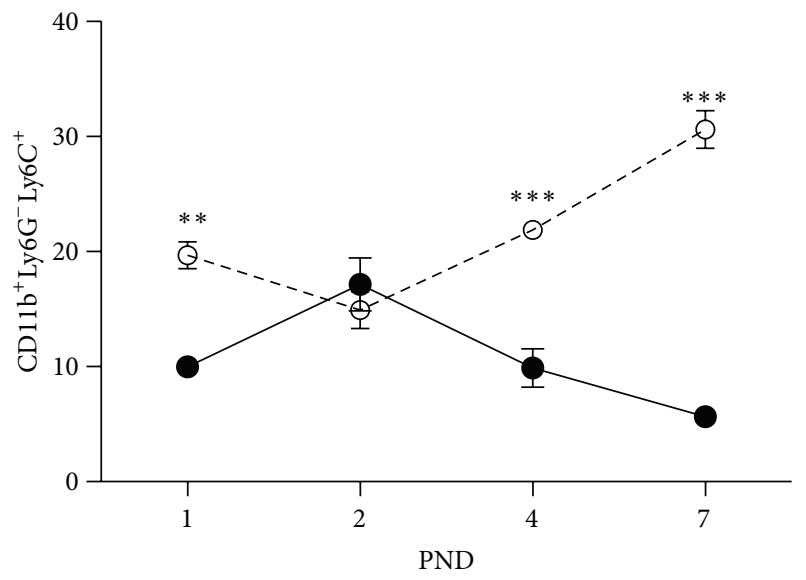

NOD

$-\Theta-\mathrm{C} 57 \mathrm{BL} / 6$

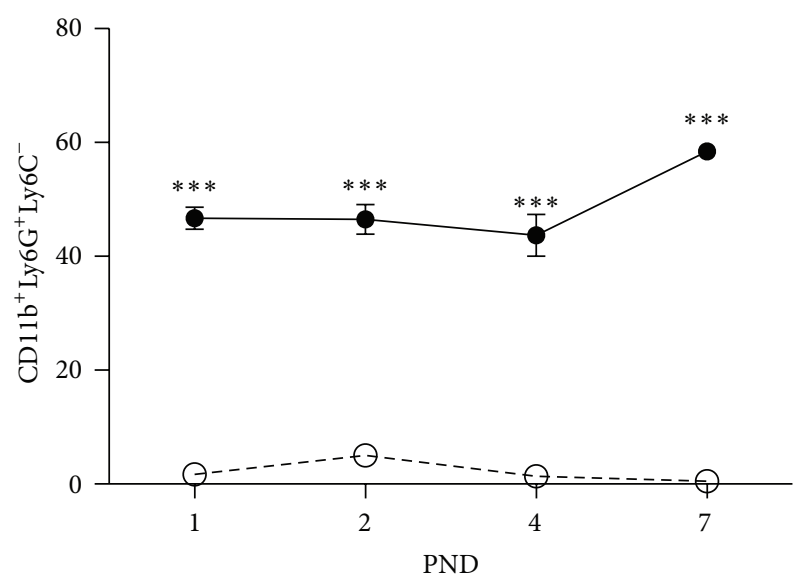

- NOD

$-\Theta-\mathrm{C} 57 \mathrm{BL} / 6$

(b)

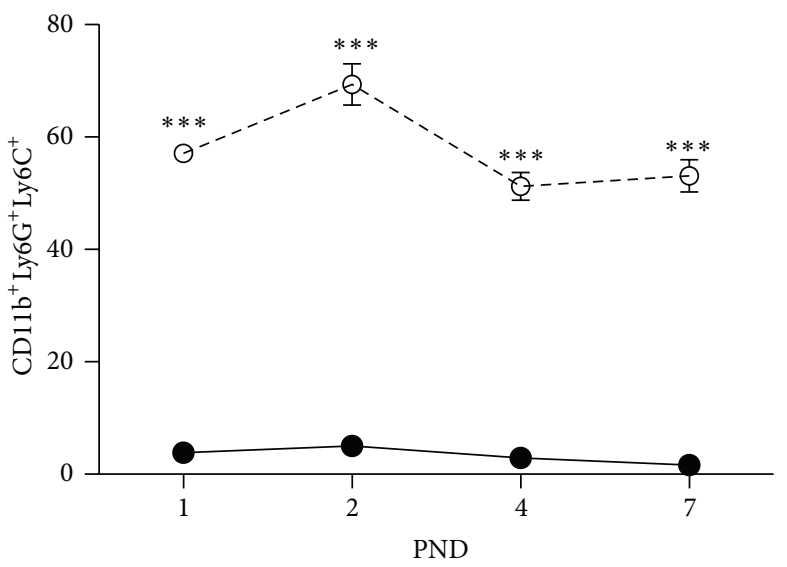

NOD

C57BL/6

(c)

(d)

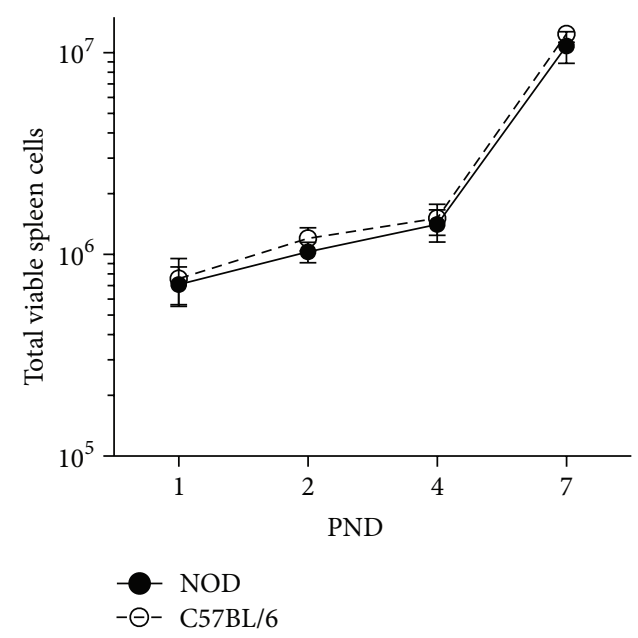

(e)

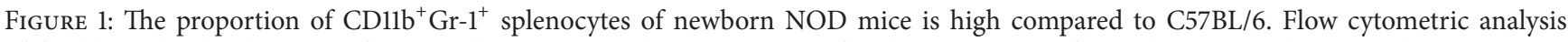
of freshly isolated splenocytes from newborn NOD and C57BL/6 mice for the expression of CD11b, Ly6C, and Ly6G. (a) Time course development of the proportion of viable CD11b ${ }^{+}$spleen cells in NOD and C57BL/6 mice at PND 1, 2, 4, and 7. Graphs (b), (c), and (d) depict gated Ly6G-Ly6C $\mathrm{C}^{+}$(d), Ly6G $\mathrm{G}^{+} \mathrm{Ly} 6 \mathrm{C}^{-}$(c), and $\mathrm{Ly}_{6 \mathrm{G}}{ }^{+} \mathrm{Ly}_{6 \mathrm{C}}^{+}$(e) double positive cells. Backgating confirmed that all three populations were $\mathrm{CD}_{11 b^{+}}$. (e) Time course of the absolute number of viable cells in spleens of NOD and C5BL/6 mice. Data presented as mean \pm SEM ( $n=8-12$ spleens). ${ }^{* *} p<0.05$. $^{* *} p<0.001$. 

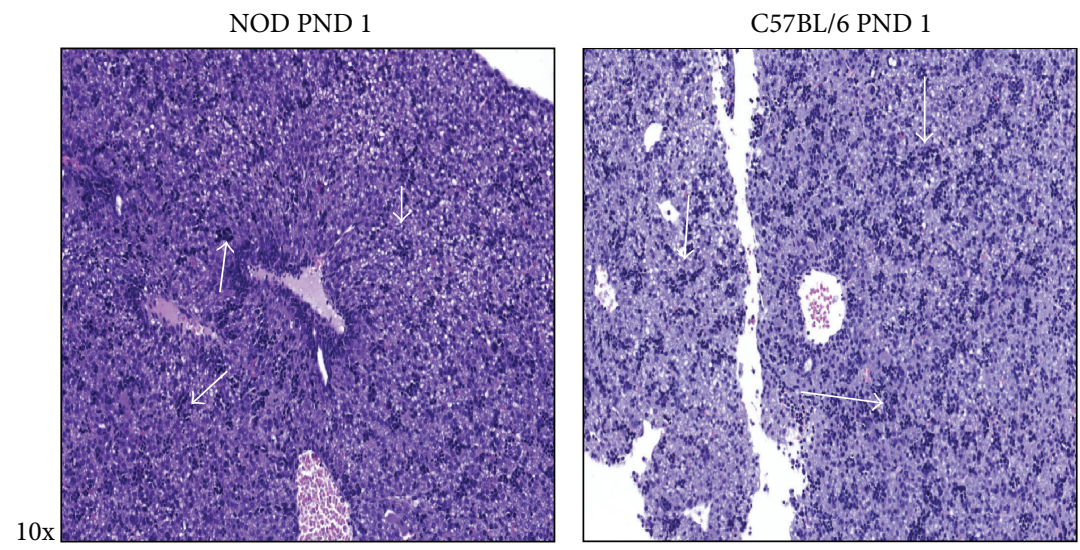

(a)

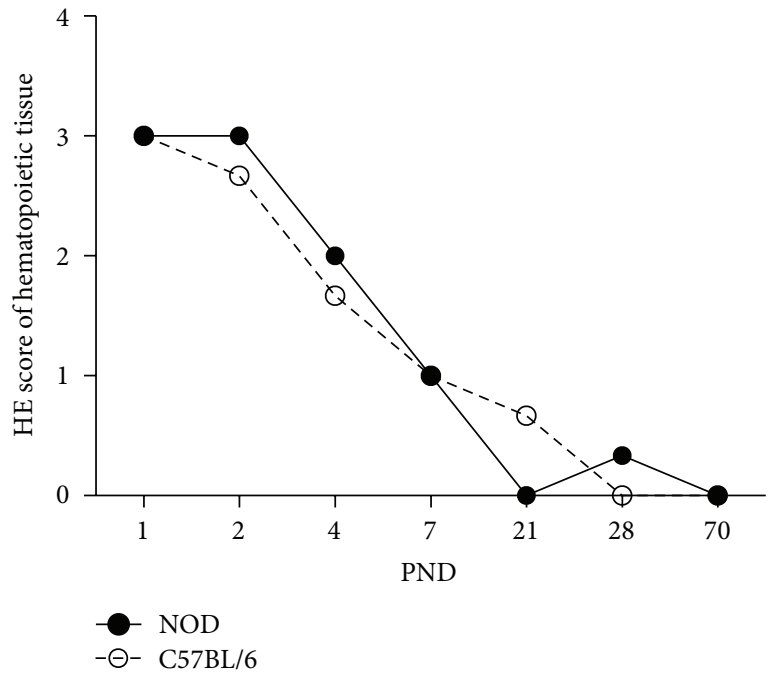

(b)

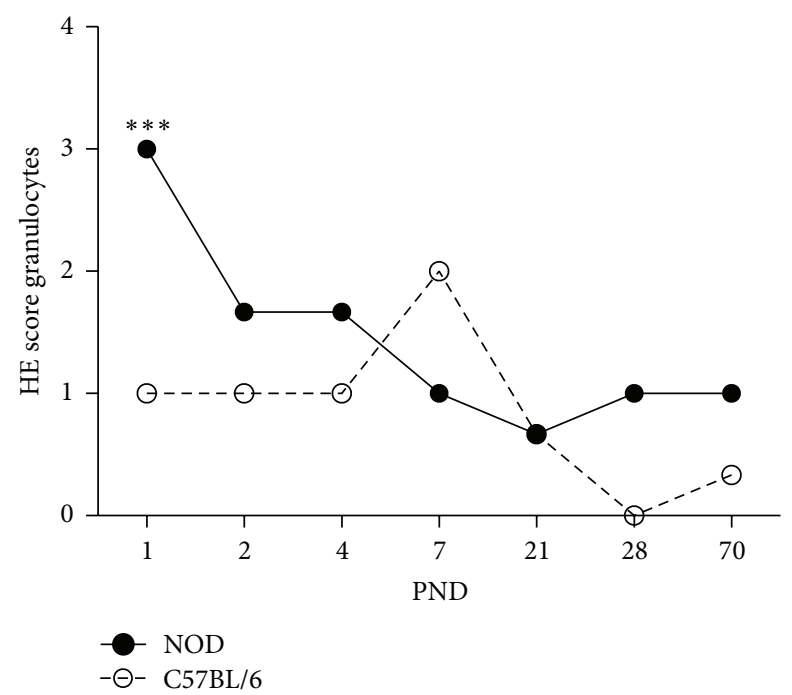

(c)

FIGURE 2: The presence of granulocytic myeloid derived cells is high in NOD mice livers. (a) Hematoxylin and eosin (HE) staining of neonatal PND 1 liver tissue sections from NOD and C57BL/6 mice. Arrows indicate hematopoietic tissue in the liver of newborn mice at PND 1. 10x magnification. (b) HE scores of hematopoietic tissue at PND 1-70 in NOD and C57BL/6 mice. Two persons evaluated HE score in a blinded fashion $(n=3)$. (c) HE score of granulocytes at PND $1-70 .{ }^{* * *} p<0.001$.

In both liver and spleen, Elane expression was significantly higher in NOD mice at PND 1-2 and PND 1-4, compared to C57BL/6 mice (Figures 4(a) and 3(b)). Arg1 expression was similar between the two strains at PND 1-4; however, Arg1 was 200x higher expressed in liver compared to spleen. In spleen at days 2 and $4, H p$ expression was higher in NOD mice (Figure 4(f)). No difference was observed in liver (Figure 4(e)), but $H p$ was expressed 50x higher in spleen than in liver, indicating that the final maturation of granulocytes takes place outside the liver.

Visual examination of spleen sections revealed that splenocytes from NOD mice had a tendency to ring-shaped rather than polylobed morphology, indicating presence of granulopoietic stage 1 myeloblasts. C57BL/6 mice had a population of cells displaying the more polylobed morphology, of more mature neutrophils (Figure 5), indicating that relatively more immature granulocytes are present in the spleen and liver of NOD mice.
3.4. Bone Marrow-Derived HSCs from NOD Mice Stimulated with Bacteria Are Able to Differentiate into Ly6C Cells. A deficiency in the coding region of Ly6C has previously been described in NOD mice [26], which may explain the low Ly6C cell profile observed in the spleen of the NOD mice. To test if bacterial stimulation of the HSCs initiates the expression of more $\mathrm{Ly}_{6} \mathrm{C}^{+}$cells, we stimulated bone marrow cells from adult NOD and C57BL/6 mice with a mixture of Gram-positive and Gram-negative bacteria and measured the expression of Ly6G and Ly6C (Figure 6). In nonstimulated cells, cells from C57BL/6 mice expressed $18.3 \%$

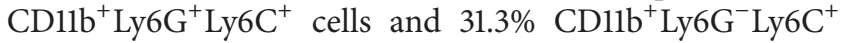

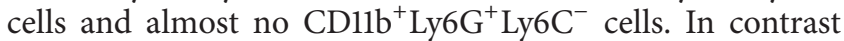
and very similar to our findings in spleen cells from new born mice, bone marrow cells from NOD mice exhibited a high proportion of Ly $6 \mathrm{G}^{+}$cells $34.0 \%$ and $8.3 \% \mathrm{Ly} 6 \mathrm{C}^{+}$. When stimulated with bacteria, bone marrow cells from NOD mice

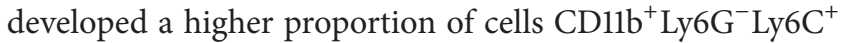



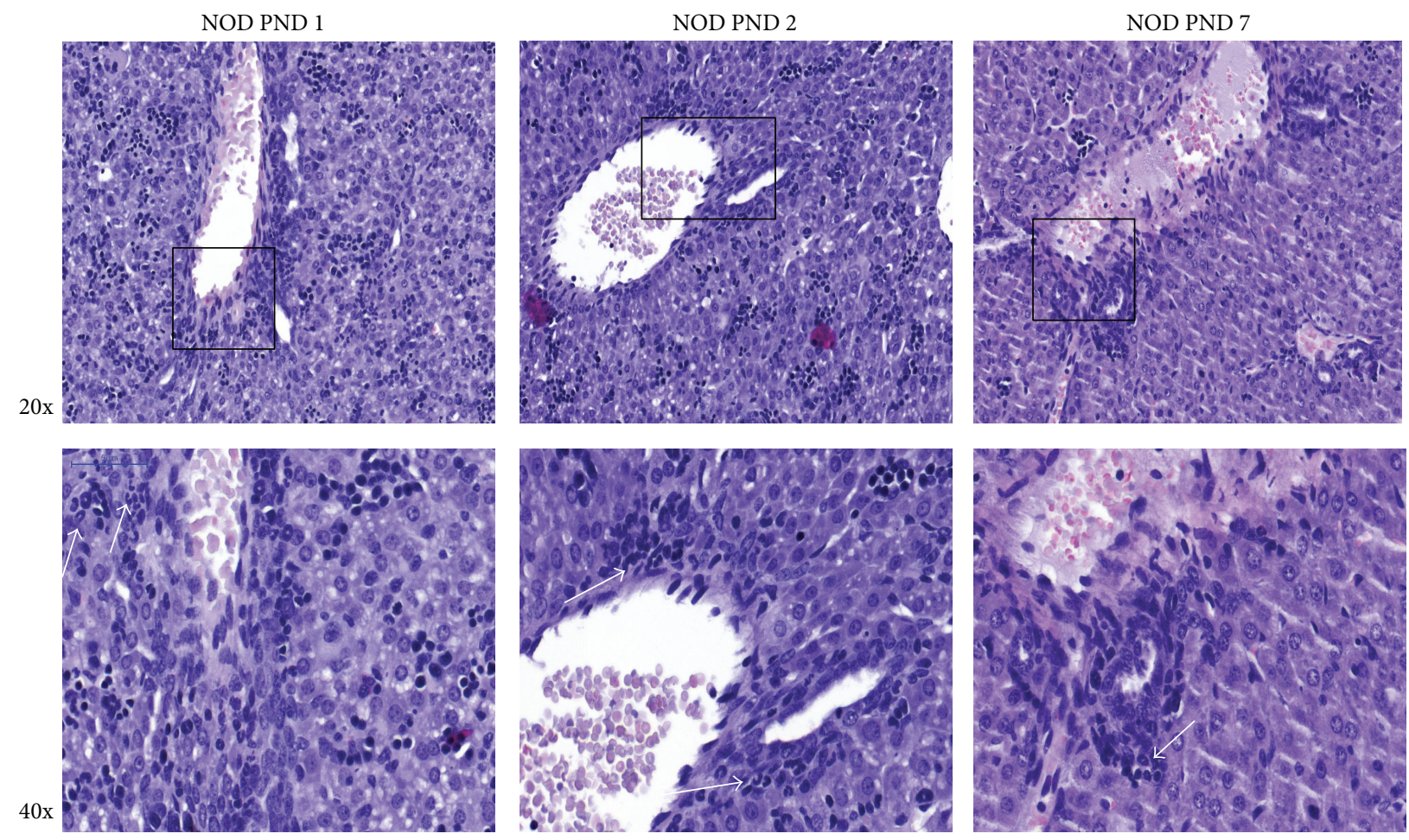

(a)
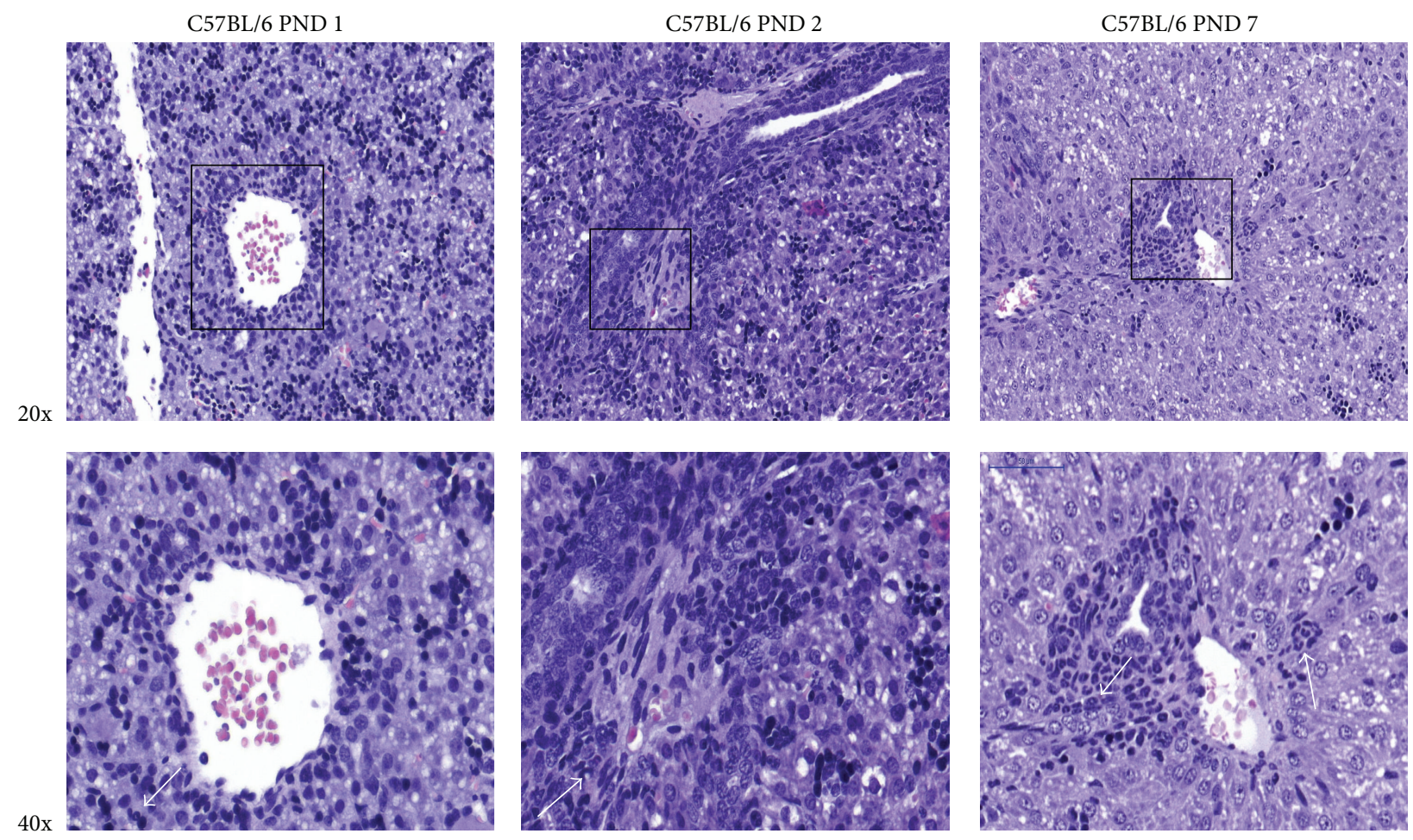

(b)

FIGURE 3: In NOD mice the granulocytic phenotype of cells accumulated around the hepatic portal veins. Hematoxylin and eosin (HE) staining of neonatal PND 1-7 liver tissue sections from NOD (a) and C57BL/6 mice (b). 

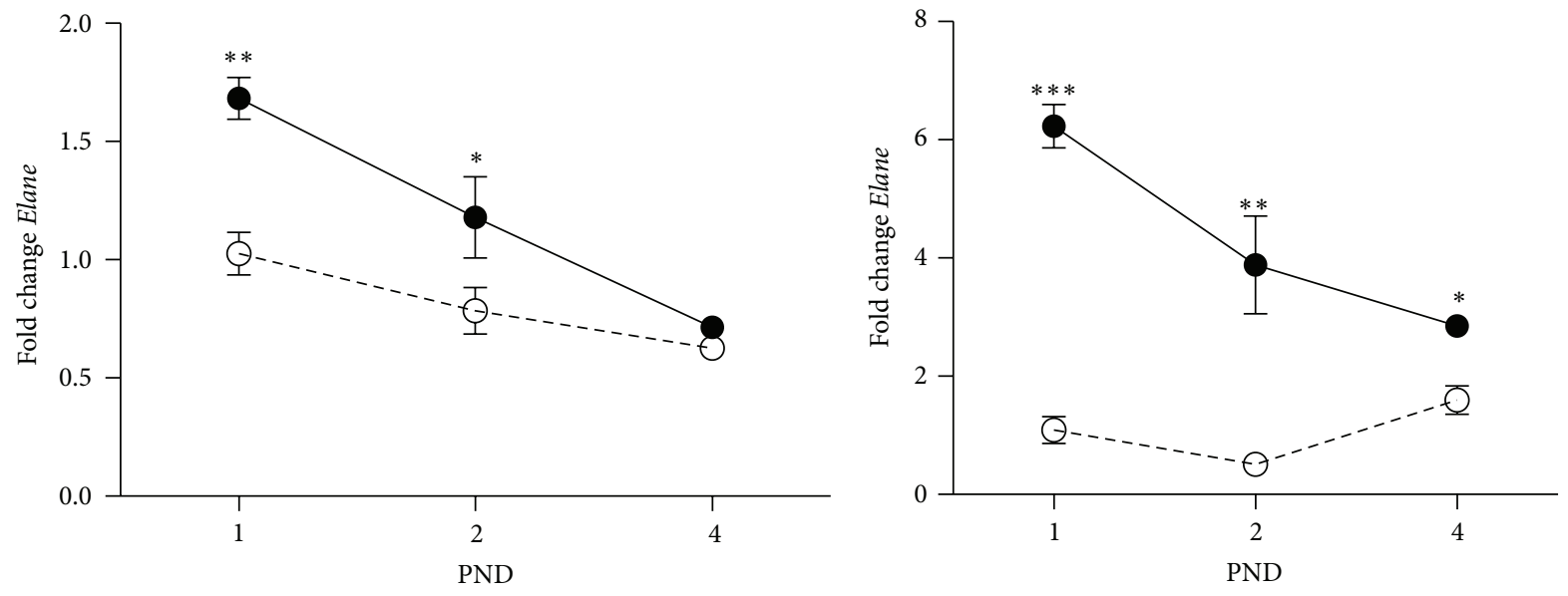

$-\mathrm{NOD}$
$-\Theta-\mathrm{C} 57 \mathrm{BL} / 6$

$\begin{array}{ll}- & \text { NOD } \\ -\odot-C 57 B L / 6\end{array}$

(a)

(b)
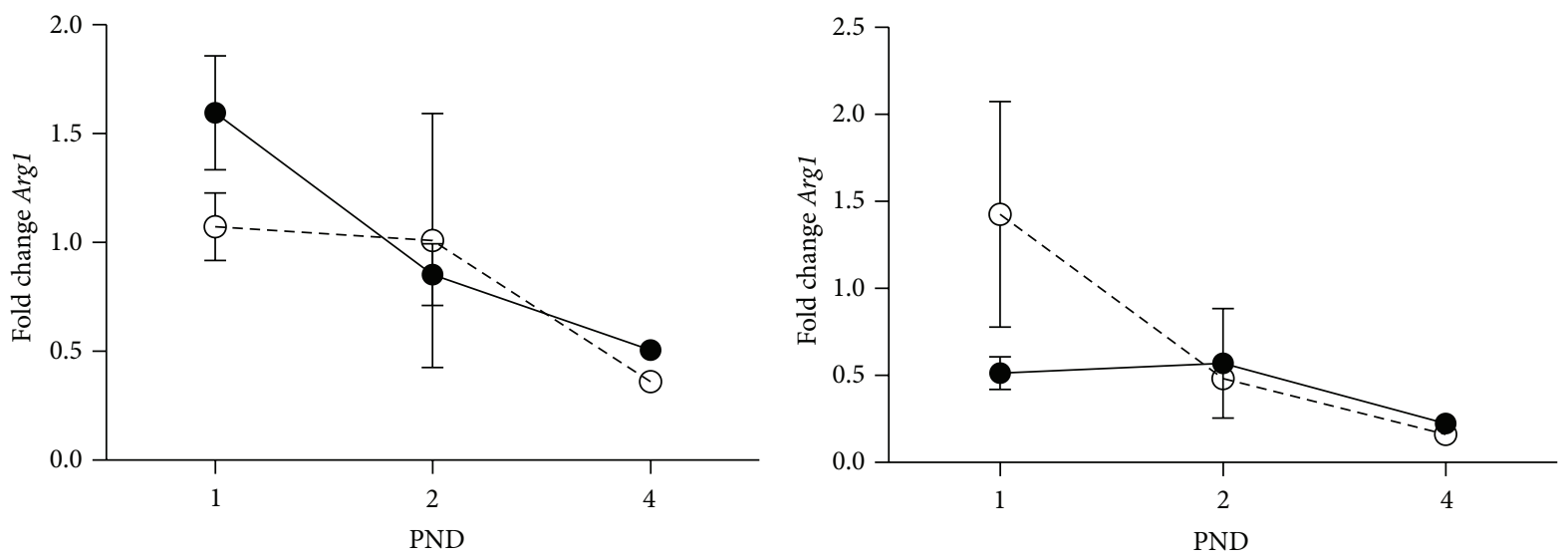

$-\mathrm{NOD}$
$-\Theta-\mathrm{C} 57 \mathrm{BL} / 6$

- NOD

(c)

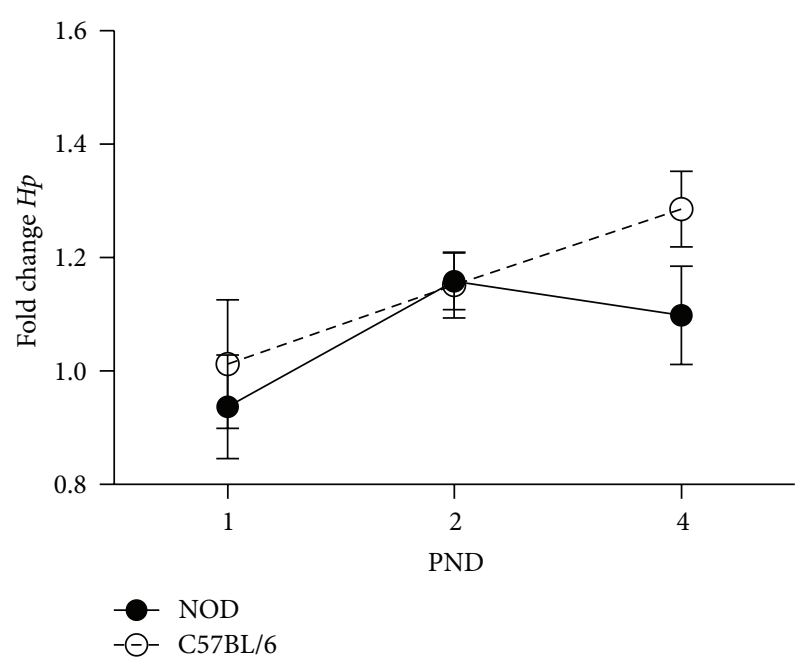

(e)

(d)

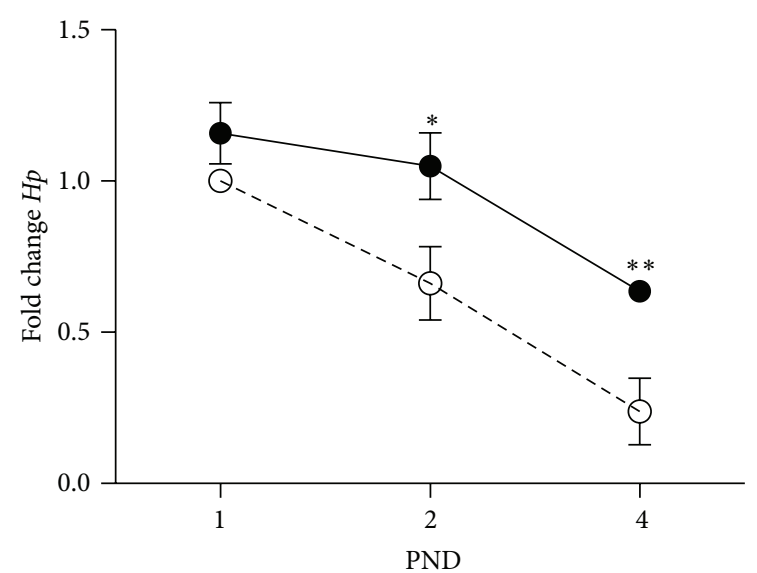

- NOD

$-\Theta-$ C57BL/6

(f)

FIGURE 4: Relative gene expression of genes expressed in differentiation of myeloid cells in liver and spleen at PND 1,2, and 4 of NOD and C57BL/6 mice. Data were normalized to Actb and then to C57BL/6 PND 1, which were defined as value 1. All samples were quantified in triplicate. All groups were included in a 2-way ANOVA. ${ }^{* * *} p<0.001, p<0.05$. (a) Expression of Elane encoding elastase in liver. (b) Expression of Elane encoding elastase in spleen. (c) Expression of Arg1 encoding arginase in liver. (d) Expression of Arg1 encoding arginase in spleen. (e) Expression of $H p$ encoding haptoglobin in liver. (f) Expression of $H p$ encoding haptoglobin in spleen. 

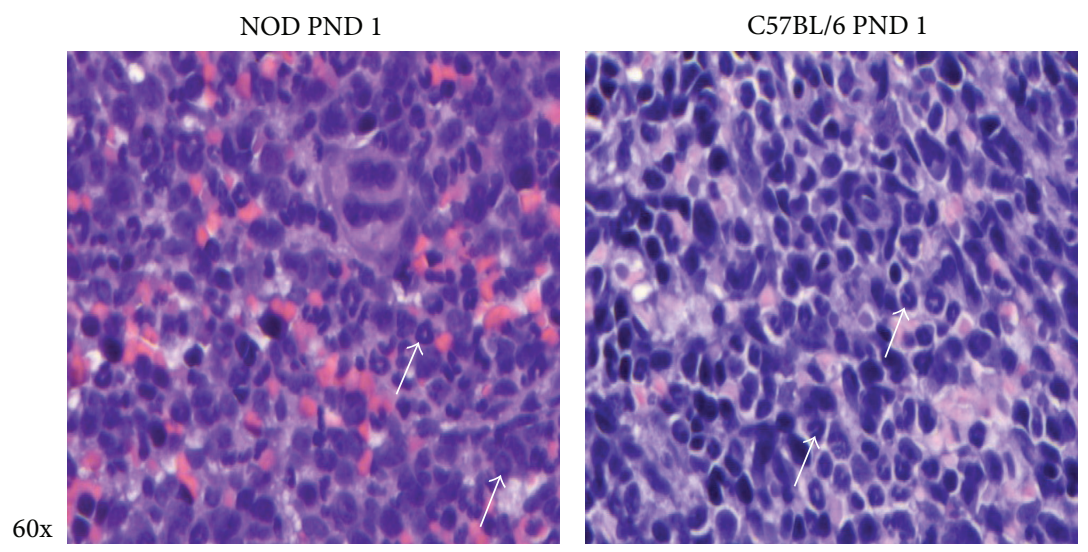

FIGURE 5: Hematoxylin and eosin (HE) staining of neonatal spleen PND 1 from NOD mice and C57BL/6. Arrows depict granulocytes at different stage of granulopoiesis. 60x magnification.
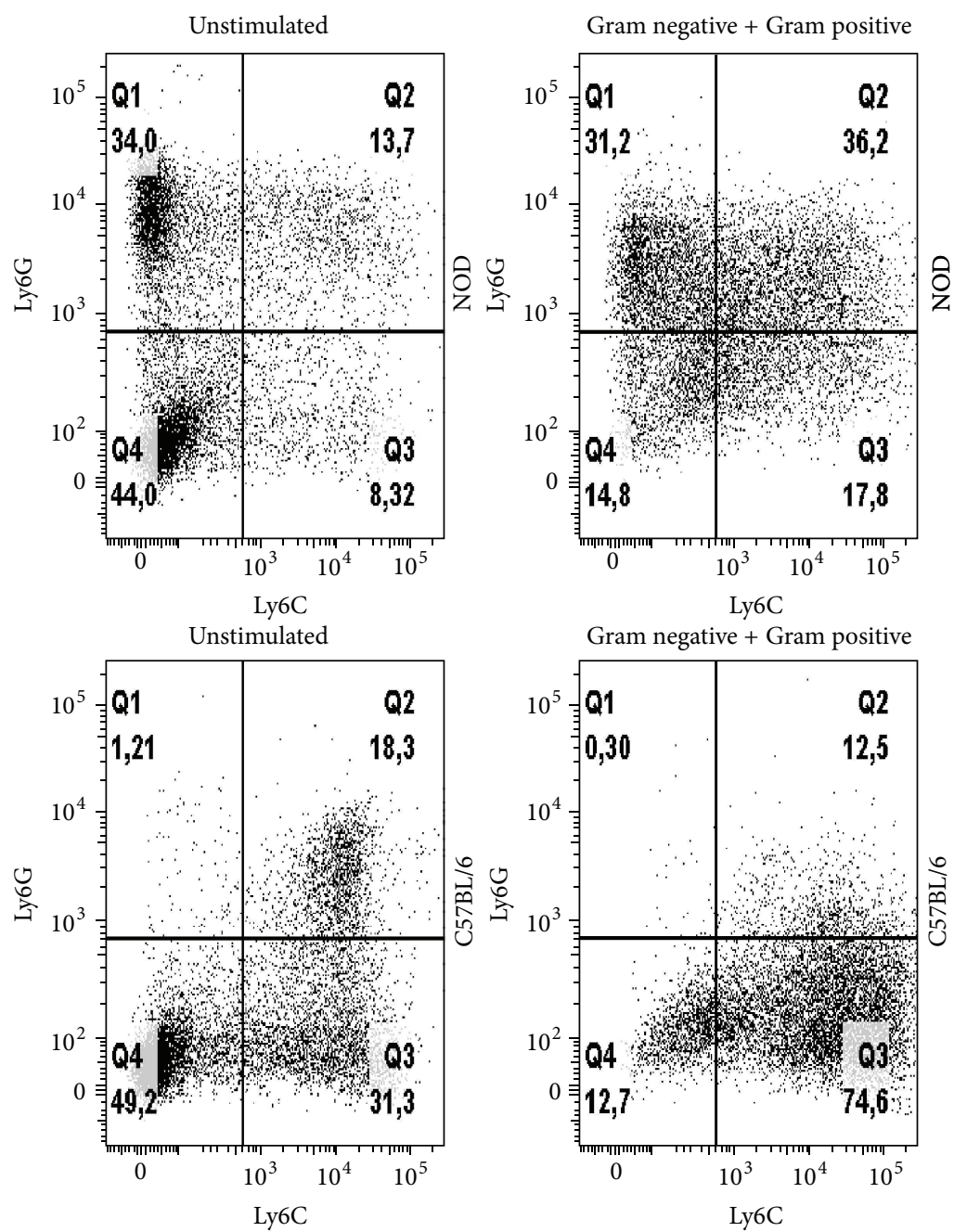

FIGURE 6: Bacterial stimulation of cultured bone marrow cells from NOD and C57BL/6 mice. On day 3 of in vitro culturing of bone marrow cells from NOD and C57BL/6 mice, mix of cells was stimulated as Gram-positive and Gram-negative bacteria (each MOI 0,5). On day 6 the cultured cells were harvested and analysed by flow cytometry. All CD11b ${ }^{+}$cells were defined positive or negative for Ly6C and Ly6G. Plots are representative for 1 of 4 experiments. 


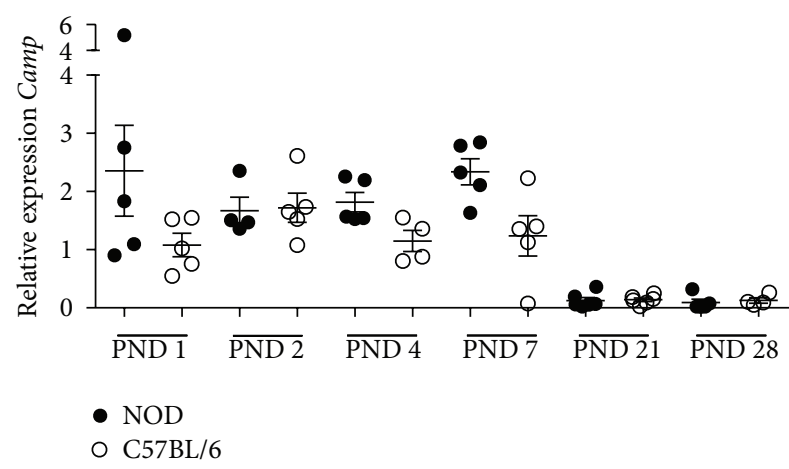

Figure 7: Relative gene expression of Camp, analyzed in intestine tissue from NOD and C57BL/6.

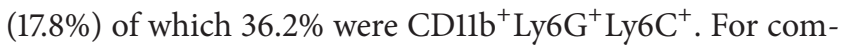
parison, cells from C57BL/6 mice changed from expressing

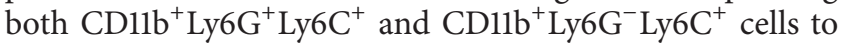

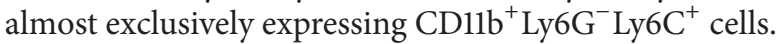

These results indicate that bacterial stimulation may overcome the genetic predisposition, affecting the expression of Ly6C.

3.5. Higher Expression of Reg3g in the Intestine of Neonatal NOD Mice Is Associated with a Restriction of Surface Attachment of Gram-Positive Bacteria and a Low Expression of Muc2. The production of the antimicrobial cathelicidinrelated antimicrobial peptide (Camp) and Reg3g in the neonatal intestine changes during maturation [37]. Camp was equally expressed by the two strains and only before weaning (Figure 7). In contrast, Reg3g that normally is weakly produced until weaning [38] had increased expression in NOD mice at PND 2-7 compared to C57BL/6 (Figure 8(a)). Upregulation of Reg $3 g$ has been associated with an aberrant production of the secreted mucus protein Muc2 [39]. Hence, we measured the expression of $\mathrm{Muc} 2$ in the neonatal period and found that Muc2 was significantly lower from PND 27 in intestine of NOD mice (Figure 8(b)). Reg3g is proposed to primarily target Gram-positive bacteria [18] and next we examined Gram-stained sections of the intestine at PND 1-7. Presence of both Gram-positive and Gram-negative bacteria was observed in the lumen of both NOD and C57BL/6 mice (Figure 8(c)) However, a limited number of bacteria were observed close to the mucosal surface of villi in the NOD intestine and those present were mostly Gram-negative. In comparison, the C57BL/6 intestine had both Gram-positive and Gram-negative bacteria associated with the mucosal surface (Figure 8(c)).

Finally, intestinal morphology between C57BL/6 and NOD mice differed between the strains, as the intestinal villi of $\mathrm{C} 57 \mathrm{BL} / 6$ showed a more dense and thick structure compared to NOD mice (Figure 8(d)).

3.6. Higher Abundance of Staphylococcus in NOD Mice Compared to C56BL/6 Mice. To assess the gut microbiota at PND 1-7 we analyzed samples of the entire intestine of C57BL/6 and NOD mice by tag-encoded 16S rRNA gene amplicon high throughput sequencing $(6,238,023$ fully overlapping raw reads, 5,575,454 after chimera purging). Four samples with less than 50,000 reads were excluded from further analysis. Remaining samples yielded on average 179,081 reads (STD $+/-)$.

The number of observed bacterial species did not differ significantly between NOD and C57BL/6 mice (observed species NOD $=27.41 \pm 12.33, \mathrm{C} 57 \mathrm{BL} / 6=34.67 \pm 20.17$, $t=-1.2, p=0.26)$. ANOSIM analysis of uniFrac-based distance metrics revealed that the GM of the 2 mice strains did not differ significantly (unweighted $R=0.053, p=0.163$, weighted $R=0.077, p=0.094$ ). Figure 9 shows the relative distribution of bacterial genera on PND 1, 2, 4, and 7 of NOD and C57BL/ 6 mice. The relative abundance of Staphylococcus was found to be significantly higher in NOD mice compared to $\mathrm{C} 57 \mathrm{BL} / 6(p($ Bonferroni-corrected $)=0.030)($ Figure 10$)$.

\section{Discussion}

Despite increased knowledge on the pathogenesis of type 1 diabetes and the role of the immune response in the development of type 1 diabetes in NOD mice, the early stages of the disease pathology remain poorly understood. Far the most studies are done in mice three weeks of age or older, in which the focus often is on the role of $\mathrm{T}$ cells infiltrating the pancreas and connecting lymph nodes [40, 41]. The events taking place prior to and causing this infiltration are largely unknown. Activation and action of some cells of the myeloid lineage are expected to be involved in the activation and polarization of $\mathrm{T}$ cells and also in recruitment of the $\mathrm{T}$ cells to the pancreas. Consequently, it is likely that hematopoietic events involving myeloid cells and taking place in the very early postnatal period are essential for the development. The key role of the GM in the development of type 1 diabetes in NOD mice has been firmly established [42]. NOD mice housed under germ-free conditions develop type 1 diabetes while mice reared under conventional conditions were demonstrated to be partly protected from the disease $[43,44]$ and GM manipulation through antibiotics or specific diets have been found to lower the incidence of autoimmune diabetes $[6,7]$. The microbial influence on development and maturation of neonate immunity is however still only sparsely described but is likely to play a key role in the development of type 1 diabetes. Others have provided evidence that changes in the maternal microbiota during pregnancy and delivery influence the development of the immune system in NOD mice [40] and CS has been associated with increased type 1 diabetes incidence in humans [13]. Furthermore, gut bacteria were recently shown to be involved in regulation of hematopoiesis [45].

We here show that the hematopoiesis and the GM and the gut epithelium deviate in the NOD mice compared to C57BL/ 6 mice in the very first postnatal period. The neonatal expression of Reg $3 g$ and $M u c 2$ in the gut is deviating in NOD mice and this coincides with vast differences in the GM of NOD and C57BL/6 mice and is perhaps most interesting, in the number of bacteria associated with the mucosal surface. In parallel with these findings, we show that the proportions of $\mathrm{Ly}_{6 \mathrm{C}} \mathrm{C}^{+}$and $\mathrm{Ly} 6 \mathrm{G}^{+}$cells in the spleen of neonatal NOD mice deviate vastly from that of C57BL/ 6 mice, as the $\mathrm{Ly} 6 \mathrm{G}^{+}$ 

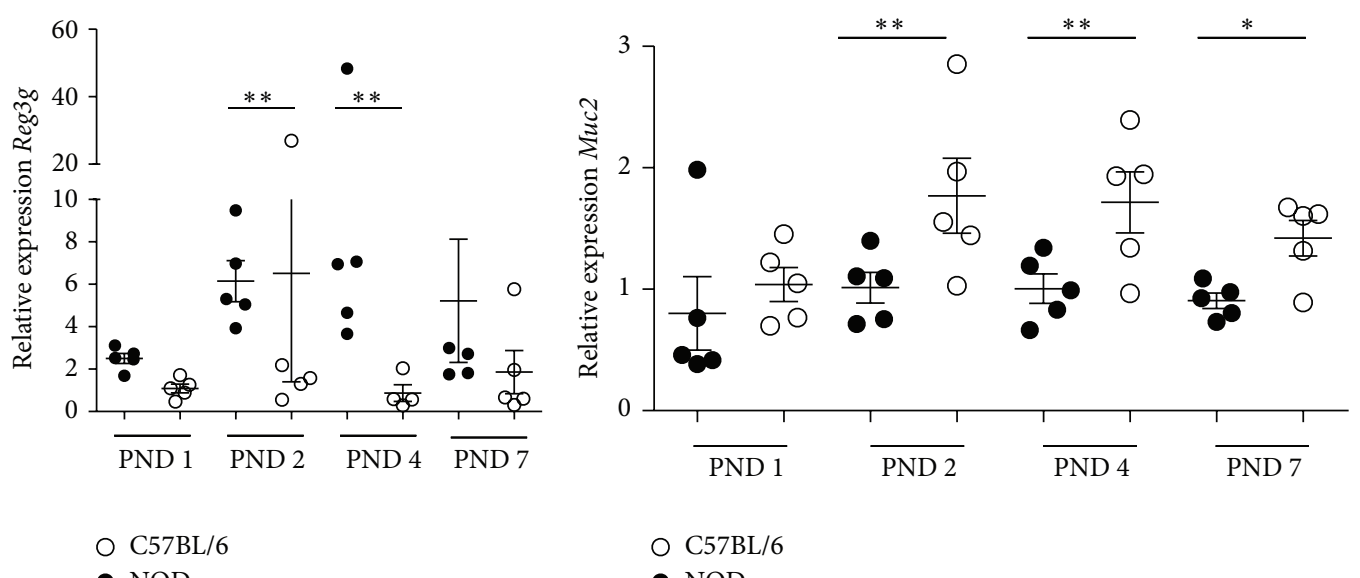

O C57BL/6

- NOD

- NOD

(a)

NOD PND 1

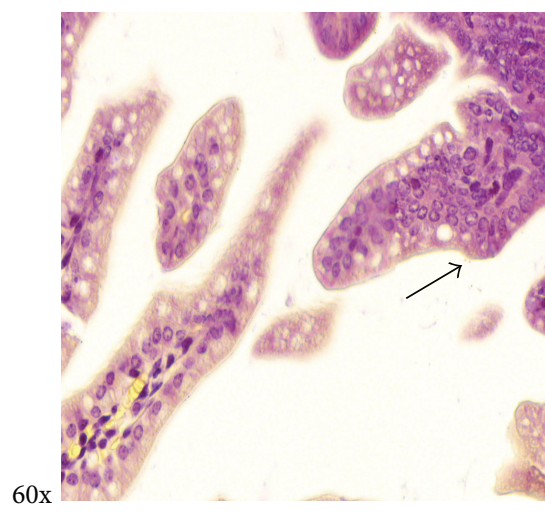

(c)

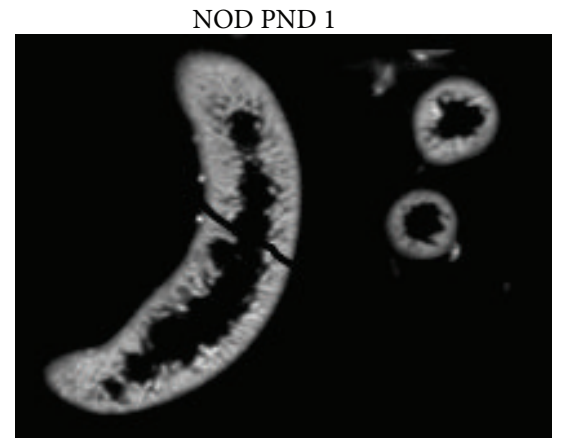

(b)

C57BL/6 PND 1

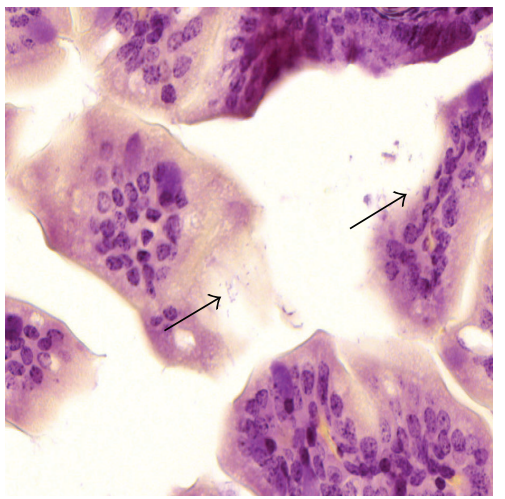

C57BL/6 PND 1

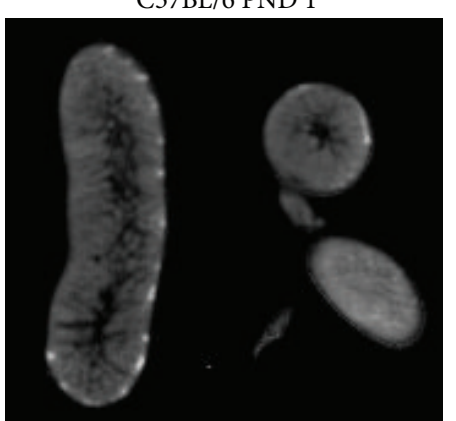

(d)

FIGURE 8: Relative gene expression of RegIII $\gamma$ analyzed in intestine tissue from NOD and C57BL/6 mice correlates with Gram-stain sections of the same intestines and OPT scanning. Data were normalized to Actb and then to C57BL/6 PND 1, which were defined as value 1. All groups were included in a 2-way ANOVA. ${ }^{* *} p<0.05$. (a) Relative gene expression of Reg3g, analyzed in intestine tissue from NOD and C57BL/6. (b) Relative gene expression of muc2 in intestinal tissue from NOD and C57BL/6. (c) Gram-stain sections of intestine 60x magnification. Arrows indicate presence of bacteria. In sections of NOD intestine (left side) bacteria were present in the lumen but very few and mostly Grampositive bacteria were attaching to the villi. In contrast a larger amount of both Gram-negative and Gram-positive bacteria was attaching to the surface of the villi in the intestine of C57BL/6 mice. (d) PND 1 mouse intestine autofluorescence image acquired with the OPT system. Digital sections of the PND 1 intestine illustrated in gray-scale demonstrating autofluorescence anatomy data in NOD and C57BL/6. Intestinal sections from C57BL/6 mice displayed more dens villi compared to NOD mice. 


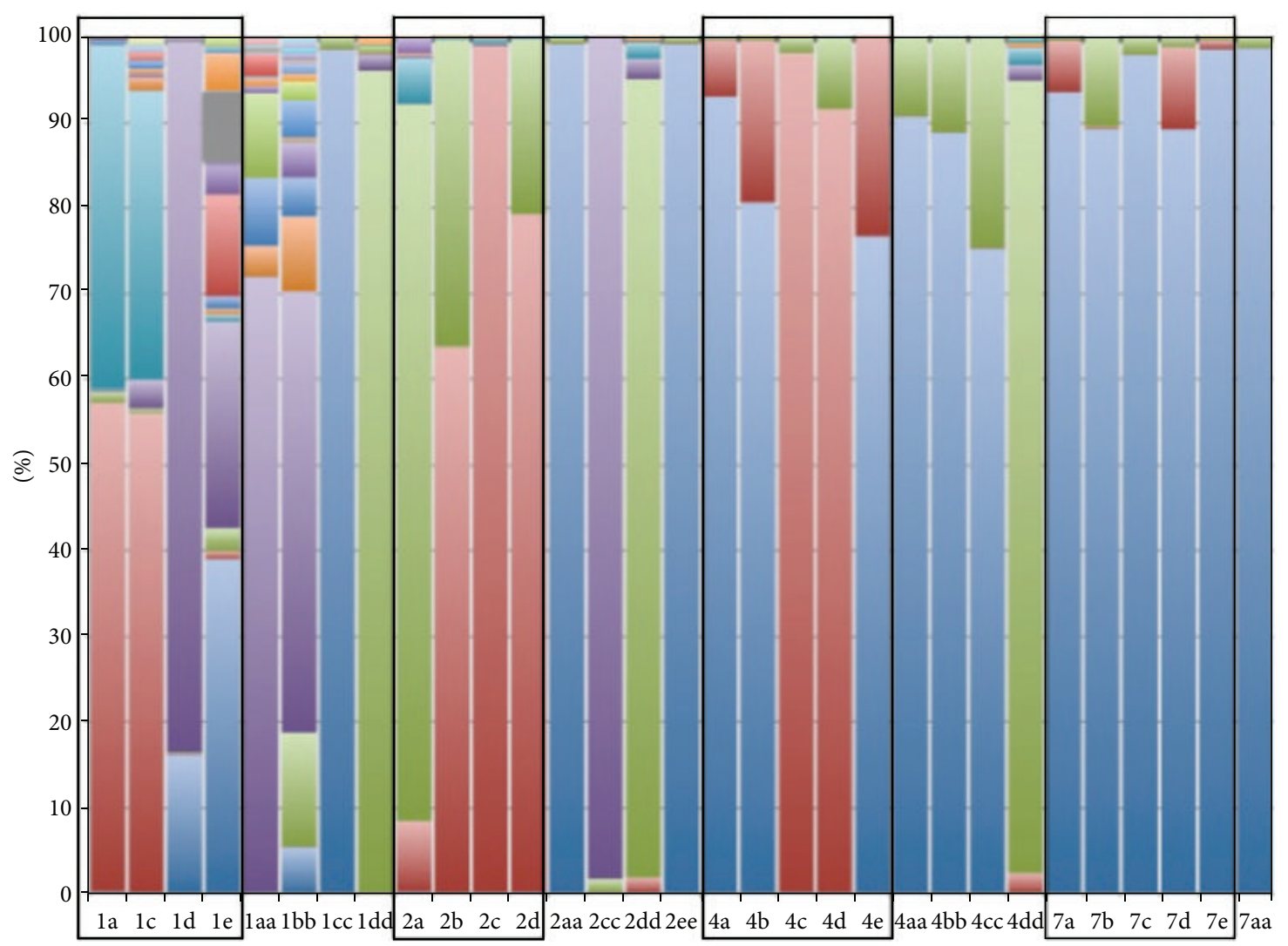

- p_Firmicutes; c_Clostridia; o_Clostridiales; f_Ruminococcaceae; g_Faecalibacterium

- p_Bacteroidetes; c_Flavobacteriia; o_Flavobacteriales; f_Flavobacteriaceae; g_Flavobacterium

- P_Firmicutes; c_Clostridia; o_Clostridiales; f_; g

p_Actinobacteria; c_Actinobacteria; o_Actinomycetales; f_Brevibacteriaceae; g_Brevibacterium

P_Bacteroidetes; c_Bacteroidia; o_Bacteroidales; f_Porphyromonadaceae; g_Paludibacter

p_Actinobacteria; c_Actinobacteria; o_Actinomycetales; f_Corynebacteriaceae; g_Corynebacterium

p_Firmicutes; c_Clostridia; o_Clostridiales; f_Veillonellaceae; g_Phascolarctobacterium

- P_Proteobacteria; c_Alphaproteobacteria; o_Rickettsiales; $f \_m i t o c h o n d r i a ; O t h e r$

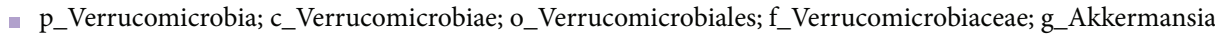

p_Firmicutes; c_Clostridia; o_Clostridiales; f_Lachnospiraceae; g_Blautia

- p_Proteobacteria; c_Gammaproteobacteria; o_Alteromonadales; $\mathrm{f} \_[\text {Chromatiaceae]; g_Rheinheimera }$

- p_Firmicutes; c_Clostridia; o_Clostridiales; f_Clostridiaceae; g-

- p_Firmicutes; c_Clostridia; o_Clostridiales; f_Lachnospiraceae; g_

p_Firmicutes; c_Clostridia; o_Clostridiales; f_Ruminococcaceae; g_

- p_Cyanobacteria; c_Chloroplast; o_Stramenopiles; f_; g

- P_Firmicutes; c_Clostridia; o_Clostridiales; f_Veillonellaceae; g_Veillonella

- P_Firmicutes; c_Clostridia; o_Clostridiales; f_Veillonellaceae; g_Dialister

- p_Proteobacteria; c_Gammaproteobacteria; o_Pseudomonadales; f_Pseudomonadaceae; g_Pseudomonas

- P_Proteobacteria; c_Gammaproteobacteria; o_Xanthomonadales; f_Xanthomonadaceae; g_

- P_Actinobacteria; c_Actinobacteria; o_Actinomycetales; f_Micrococcaceae; g_

- P_Proteobacteria; c_Gammaproteobacteria; o_Xanthomonadales; f_Xanthomonadaceae; g_Stenotrophomonas

- P_Proteobacteria; c_Gammaproteobacteria; o_Aeromonadales; f_Succinivibrionaceae; g_Succinivibrio

- P_Fusobacteria; c_Fusobacteriia; o_Fusobacteriales; f_Fusobacteriaceae; g_Fusobacterium

- P_Bacteroidetes; c_Bacteroidia; o_Bacteroidales; f_Bacteroidaceae; g_Bacteroides

- p_Bacteroidetes; c_Bacteroidia; o_Bacteroidales; f_Prevotellaceae; g_Prevotella

- P_Firmicutes; c_Bacilli; o_Bacillales; f_Planococcaceae; $g$

- P_Proteobacteria; c_Gammaproteobacteria; o_Enterobacteriales; f_Enterobacteriaceae; g_

- p_Firmicutes; c_Bacilli; o_Lactobacillales; f_Streptococcaceae; g_Streptococcus

- p_Firmicutes; c_Bacilli; o_Bacillales; f_Staphylococcaceae; g_Staphylococcus

- p_Firmicutes; c_Bacilli; o_Lactobacillales; f_Lactobacillaceae; g_Lactobacillus

FIGURE 9: Microbiota composition of the intact intestine from C57BL/6 and NOD mice at PND 1, 2, and 4 as determined by 165 rRNA gene amplicon high throughput sequencing. Brackets indicate phylum. Red: Staphylococcus ( $g$ ) (Firmicutes). Blue: Lactobacillus ( $g$ ) (Firmicutes). Purple: Enterobacteriaceae $(f)$ (Proteobacteria). Green: Streptococcus ( $g$ ) (Firmicutes). Turquoise: Planococcaceae ( $f$ ) (Firmicutes). Black boxes indicate NOD mice. 


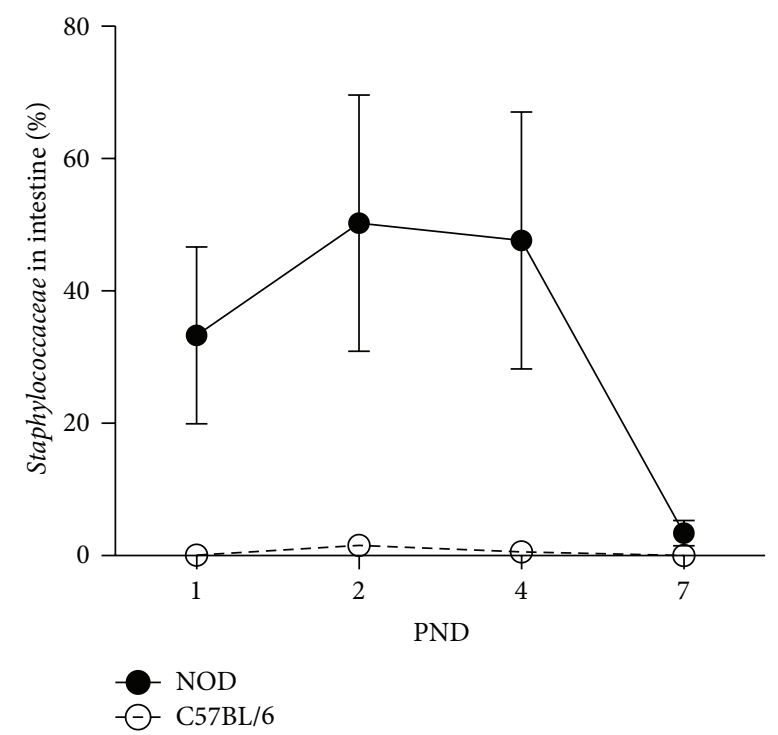

FIgURE 10: Microbiota composition of the intact intestine from C57BL/6 and NOD mice at PND 1, 2, and 4 as determined by 16S rRNA gene amplicon high throughput sequencing. Presence of Staphylococcus in the intestine of NOD and C57BL/6 mice. Data are expressed as percentage of bacteria present in intestine. Data are mean \pm SEM ( $n=4-5$ intestines).

cells dominate and Ly6C $\mathrm{C}^{+}$cell number is very low in NOD mice, but this can, at least in vitro, partly be circumvented by bacterial stimulation.

At birth the spleen and the liver constitute major hematopoietic tissues in mice and may represent important sites for encounters between microorganisms and immune system. Puga et al. found granulocytic infiltration of perimarginal-zones of the spleen after postnatal mucosal colonization by microbes, showed the presence of bacterial $16 \mathrm{~s}$ ribosomal RNA in these areas in the spleen, and found that the granulocytic phenotype was dependent on the microbiota [46]. Hence, the presence of bacteria or microbial components in the spleen (and liver) may influence the hematopoiesis here.

In the present study we demonstrate that spleen cells at PND 1-7 in NOD mice comprise a large population of immature granulocytic cells expressing $\mathrm{CD}_{11 b^{+}} \mathrm{Ly}_{6 \mathrm{G}}{ }^{+} \mathrm{Ly} 6 \mathrm{C}^{-}$, while in $\mathrm{C} 57 \mathrm{BL} / 6$ the far largest proportion of $\mathrm{CD}_{11 \mathrm{~b}^{+}}$cells were Ly $6 \mathrm{C}^{+} \mathrm{Ly} 6 \mathrm{G}^{+}$cells.

The lack of Ly6C expression in the bone marrow cells of NOD mice is long known $[23,26]$ and has been suggested to influence the propensity to development of type 1 diabetes, but the mechanism behind how these deviating myeloid cells influence disease development is largely unknown. In vitro we showed that bone marrow cells from NOD mice exhibit a similar Ly6 $\mathrm{C}^{-}$cell profile as the spleen cells of newborn mice, but these could be prompted to change to Ly6C expressing cells by the addition of bacteria. Thus, the presence of bacteria or microbial epitopes may have larger influence on a proper immune development in NOD mice compared to other, nondiabetic mouse strains.
Our microscopy data from NOD mice liver showed a larger number of granulocytic cells than in livers of C57BL/6 mice and abundant granulocyte like cells accumulated around the hepatic veins although the same amount of hematopoietic tissue present in NOD and C57BL/6 mice from PND 1. This might indicate either an earlier efflux of granulocytes from livers of C57BL/6 or a larger number of granulocytes are formed in the NOD mice. A postnatal efflux of hematopoietic cells from liver to spleen has previously been demonstrated in mice [47] and we showed here that in spleen granulocytic cells in early (Elane expressing) as well as late (Hp expressing) differential state are present while, in liver, primarily early differential state granulocytes are present, supporting the possibility that many immature granulocytes migrate from liver to spleen. Also, in spleen both Elane and $\mathrm{H} p$ were more highly expressed in NOD mice and in the liver only Elane was higher expressed furthermore indicative of more granulocytic cells in these organs in NOD mice.

It is well established that, during the first postnatal weeks, Paneth cells are absent in the crypts of the mouse intestine and in this period the intestinal epithelium expresses Camp [37]. As expected, the expression of Camp decreased around the time of weaning and, at this time (PND 28), mature Paneth cells producing Reg3g are present. However, other cells such as goblet cells and enterocytes have also been demonstrated to express Reg3g [48] and are likely producers of this defensin at this early stage. In contrast, the expression of $M u c 2$ was significantly lower in NOD mice compared to C57BL/6 mice. We have earlier shown that the presence of a full GM in the postnatal period leads to upregulation in the gene transcription of Muc2 [49]. In the present study we found that a larger number of bacteria were associated with the mucosa of C57BL/6 mice compared to NOD mice, which could be speculated to be the cause of the increased Muc2 expression. A lower production of Muc2 would likely influence the composition of the microbiota, as glycans of mucin functions as source of nutrients for many bacteria [50]. Paassen et al. [48] have recently demonstrated that absence of $M u c 2$ was followed by upregulation of Reg $3 g$ in the intestine of muc2 $^{-/-}$mice. Thus, the low expression of $M u c 2$ could explain the upregulated level of Reg3g in the NOD mice. When inspecting the morphology of the intestine of the newborn mice by OPT scanning, we observed considerable morphological differences between PND 1 NOD and C57BL/6 mice, as NOD mice intestine showed a thinner and less dense structure. Hence, physiological differences as well as differences in gene expression which may affect translocation of bacteria or microbial components from the gut were seen.

Reg3g binds peptidoglycan [18], expressed on the surface of Gram-positive bacteria. Our Gram-staining analysis revealed both Gram-negative and Gram-positive bacteria in the lumen of both mice strains. While both classes of bacteria were attached to the surface of the villi in the C57BL/6 mice, few Gram-negative bacteria were attached in NOD mice intestine. Interestingly, a bacteria profile enriched in Staphylococcus was prevailing in the intestine of NOD mice at PND 1-4. This genus of Gram-positive bacteria has previously 
been associated with children delivered by caesarean section and with an increased risk of developing type 1 diabetes [51]. The very different and less diverse bacterial composition found in newborn NOD mice is likely to influence gene expression in the gut and thus factors such as the mucus thickness. Hildebrand et al. reported that genetics directs $20 \%$ of the GM composition [52] and genetics in the gut epithelium may also be involved in our study, as the animals were reared in the same animal facility and handled by the same persons, giving different basis for colonisation of bacteria. Interestingly, the high level of Staphylococci found at PND 1-4 in NOD mice decreased dramatically at PND 7, which is in correspondence with the expression level of Reg3g. Whether Staphylococci are directly involved in the expression of $\operatorname{Reg} 3 g$ is however purely speculative.

In summary, we here demonstrate huge differences in the hematopoiesis, in GM, and in gut epithelium between NOD and C57BL/6 mice during the very first postnatal period. We propose that these differences might be involved in the predisposition for developing type 1 diabetes in the NOD mice.

\section{Highlights}

(i) Hematopoiesis deviates in the NOD mice compared to $\mathrm{C} 57 \mathrm{BL} / 6$ mice in the postnatal period.

(ii) Neonatal expression of Reg3g and Muc2 in the gut is deviating in NOD mice.

(iii) Fewer bacteria attach to the intestinal surface in neonatal NOD.

(iv) In vitro microbial stimulation of bone marrow cells leads to changes in hematopoiesis.

\section{Conflict of Interests}

The authors declare no conflict of interests.

\section{Authors' Contribution}

Dina Silke Malling Damlund conceived and designed the study, analyzed histology, performed flow cytometry, analyzed data, contributed to the discussion, and wrote the paper. Hanne Frøkiær was involved in designing the study, contributed to the discussion, and wrote the paper. Stine Broeng Metzdorff was involved in designing the study, the analysis of gene expression data, performed and analyzed OPT, contributed to the discussion, and wrote the paper. Jane Preuss Hasselby performed and analyzed histology. Karsten Stig Buschard contributed to the discussion. Maria Wiese performed and analyzed pyrosequencing. Dennis Sandris Nielsen analyzed pyrosequencing and contributed to the discussion. Mia Lundsager performed the in in vitro bone marrow stimulation. Axel Kornerup Hansen was involved in designing the study and contributing to the discussion. Dina Silke Malling Damlund is the guarantor of the work and as such had full access to all the data in the study and takes responsibility for the integrity of the data and the accuracy of the data analysis.

\section{Acknowledgments}

M. Petersen, Faculty of Faculty of Health and Medical Sciences, University of Copenhagen, Denmark, is kindly acknowledged for helping with the qPCR work. H. Farlov, Faculty of Health and Medical Sciences, University of Copenhagen, Denmark, is kindly acknowledged for taking care of the mice. H. Calum, Hvidovre Hospital, Denmark, is kindly acknowledged for helping with the microbiology. Nina Sonne is acknowledged for helping with DNA extraction. This work was funded by The Neomune Centre financed by The Danish Council for Strategic Research and a mobility stipend financed by the Danish Research Council.

\section{References}

[1] T. Fujita, R. Yui, Y. Serizawa, S. Makino, and Y. Tochino, "Lymphocytic insulitis in a nonobese diabetic (Nod) strain of micean immunochemical and electron-microscope investigation," Biomedical Research, vol. 3, pp. 429-443, 1982.

[2] S. Makino, K. Kunimoto, Y. Muraoka, Y. Mizushima, K. Katagiri, and Y. Tochino, "Breeding of a non-obese, diabetic strain of mice," Experimental Animals, vol. 29, no. 1, pp. 1-13, 1980.

[3] D. P. Funda, A. Kaas, T. Bock, H. Tlaskalová-Hogenová, and K. Buschard, "Gluten-free diet prevents diabetes in NOD mice," Diabetes/Metabolism Research and Reviews, vol. 15, no. 5, pp. 323-327, 1999.

[4] C. King and N. Sarvetnick, "The incidence of type-1 diabetes in NOD mice is modulated by restricted flora not germ-free conditions," PLoS ONE, vol. 6, no. 2, Article ID e17049, 2011.

[5] L. S. Wicker, J. A. Todd, and L. B. Peterson, "Genetic control of autoimmune diabetes in the NOD mouse," Annual Review of Immunology, vol. 13, pp. 179-200, 1995.

[6] C. H. F. Hansen, Ł. Krych, K. Buschard et al., "A maternal gluten-free diet reduces inflammation and diabetes incidence in the offspring of NOD Mice," Diabetes, vol. 63, no. 8, pp. 28212832, 2014

[7] C. H. F. Hansen, L. Krych, D. S. Nielsen et al., "Early life treatment with vancomycin propagates Akkermansia muciniphila and reduces diabetes incidence in the NOD mouse," Diabetologia, vol. 55, no. 8, pp. 2285-2294, 2012.

[8] D. V. Serreze and E. H. Leiter, "Genes and cellular requirements for autoimmune diabetes susceptibility in nonobese diabetic mice," Current Directions in Autoimmunity, vol. 4, pp. 31-67, 2001.

[9] D. S. Nielsen, Ł. Krych, K. Buschard, C. H. F. Hansen, and A. K. Hansen, "Beyond genetics. Influence of dietary factors and gut microbiota on type 1 diabetes," FEBS Letters, vol. 588, no. 22, pp. 4234-4243, 2014.

[10] M. A. Kriegel, E. Sefik, J. A. Hill, H.-J. Wu, C. Benoist, and D. Mathis, "Naturally transmitted segmented filamentous bacteria segregate with diabetes protection in nonobese diabetic mice," Proceedings of the National Academy of Sciences of the United States of America, vol. 108, no. 28, pp. 11548-11553, 2011.

[11] K. Buschard, C. Pedersen, S. V. Hansen, I. Hageman, K. Aaen, and K. Bendtzen, "Anti-diabetogenic effect of fusidic acid in diabetes prone bb rats," Autoimmunity, vol. 14, no. 2, pp. 101104, 1992. 
[12] T. Matsuzaki, Y. Nagata, S. Kado et al., "Prevention of onset in an insulin-dependent diabetes mellitus model, NOD mice, by oral feeding of Lactobacillus casei," APMIS, vol. 105, no. 8, pp. 643-649, 1997.

[13] C. R. Cardwell, L. C. Stene, G. Joner et al., "Caesarean section is associated with an increased risk of childhood-onset type 1 diabetes mellitus: a meta-analysis of observational studies," Diabetologia, vol. 51, no. 5, pp. 726-735, 2008.

[14] J. J. Cebra, "Influences of microbiota on intestinal immune system development," American Journal of Clinical Nutrition, vol. 69, no. 5, pp. 1046s-1051s, 1999.

[15] J. L. Round and S. K. Mazmanian, "The gut microbiota shapes intestinal immune responses during health and disease," Nature Reviews Immunology, vol. 9, no. 5, pp. 313-323, 2009.

[16] S. Vaishnava, C. L. Behrendt, A. S. Ismail, L. Eckmann, and L. V. Hooper, "Paneth cells directly sense gut commensals and maintain homeostasis at the intestinal host-microbial interface," Proceedings of the National Academy of Sciences of the United States of America, vol. 105, no. 52, pp. 20858-20863, 2008.

[17] M. E. V. Johansson and G. C. Hansson, "Microbiology. Keeping bacteria at a distance," Science, vol. 334, no. 6053, pp. 182-183, 2011.

[18] S. Vaishnava, M. Yamamoto, K. M. Severson et al., "The antibacterial lectin RegIIIgamma promotes the spatial segregation of microbiota and host in the intestine," Science, vol. 334, no. 6053, pp. 255-258, 2011.

[19] A. E. Dorofeyev, I. V. Vasilenko, O. A. Rassokhina, and R. B. Kondratiuk, "Mucosal barrier in ulcerative colitis and crohn's disease," Gastroenterology Research and Practice, vol. 2013, Article ID 431231, 9 pages, 2013.

[20] H. Ogawa, K. Fukushima, H. Naito et al., "Increased expression of HIP/PAP and regenerating gene III in human inflammatory bowel disease and a murine bacterial reconstitution model," Inflammatory Bowel Diseases, vol. 9, no. 3, pp. 162-170, 2003.

[21] S. Ikehara, H. Ohtsuki, R. A. Good et al., "Prevention of type I diabetes in nonobese diabetic mice by allogeneic bone marrow transplantation," Proceedings of the National Academy of Sciences of the United States of America, vol. 82, no. 22, pp. 7743$7747,1985$.

[22] H. Li, C. L. Kaufman, S. S. Boggs, P. C. Johnson, K. D. Patrene, and S. T. Ildstad, "Mixed allogeneic chimerism induced by a sublethal approach prevents autoimmune diabetes and reverses insulitis in nonobese diabetic (NOD) mice," The Journal of Immunology, vol. 156, no. 1, pp. 380-388, 1996.

[23] P. B. Langmuir, M. M. Bridgett, A. L. M. Bothwell, and I. N. Crispe, "Bone marrow abnormalities in the non-obese diabetic mouse," International Immunology, vol. 5, no. 2, pp. 169-177, 1993.

[24] P. Y. Lee, J.-X. Wang, E. Parisini, C. C. Dascher, and P. A. Nigrovic, "Ly6 family proteins in neutrophil biology," Journal of Leukocyte Biology, vol. 94, no. 4, pp. 585-594, 2013.

[25] S. Yamanouchi, K. Kuwahara, A. Sakata et al., "A T cell activation antigen, Ly6C, induced on CD4+ Th1 cells mediates an inhibitory signal for secretion of IL-2 and proliferation in peripheral immune responses," European Journal of Immunology, vol. 28, no. 2, pp. 696-707, 1998.

[26] W. M. Philbrick, S. E. Maher, M. M. Bridgett, and A. L. M. Bothwell, "A recombination event in the 5 ' flanking region of the Ly-6C gene correlates with impaired expression in the NOD, NZB and ST strains of mice," The EMBO Journal, vol. 9, no. 8, pp. 2485-2492, 1990.
[27] C. H. F. Hansen, H. Frøkiær, A. G. Christensen et al., "Dietary xylooligosaccharide downregulates IFN $-\gamma$ and the low-grade inflammatory cytokine IL- $1 \beta$ systemically in mice," The Journal of Nutrition, vol. 143, no. 4, pp. 533-540, 2013.

[28] K. J. Livak and T. D. Schmittgen, "Analysis of relative gene expression data using real-time quantitative PCR and the 2(Delta Delta C(T)) method," Methods, vol. 25, pp. 402-408, 2001.

[29] B. Pyndt Jørgensen, J. T. Hansen, L. Krych et al., "A possible link between food and mood: dietary impact on gut microbiota and behavior in BALB/c mice," PLoS ONE, vol. 9, no. 8, Article ID e103398, 2014.

[30] R. C. Edgar, "UPARSE: highly accurate OTU sequences from microbial amplicon reads," Nature Methods, vol. 10, no. 10, pp. 996-998, 2013.

[31] H. R. Christensen, H. Frøkiær, and J. J. Pestka, "Lactobacilli differentially modulate expression of cytokines and maturation surface markers in murine dendritic cells," The Journal of Immunology, vol. 168, no. 1, pp. 171-178, 2002.

[32] J. Bancroft and M. Gamble, Theory and Practice of Histological Techniques, Churchill Livingstone, Philadelphia, Pa, USA, 2008.

[33] T. Alanentalo, A. Asayesh, H. Morrison et al., "Tomographic molecular imaging and 3D quantification within adult mouse organs," Nature Methods, vol. 4, no. 1, pp. 31-33, 2007.

[34] M. Faurschou and N. Borregaard, "Neutrophil granules and secretory vesicles in inflammation," Microbes and Infection, vol. 5, no. 14, pp. 1317-1327, 2003.

[35] K. Theilgaard-Mönch, L. C. Jacobsen, M. J. Nielsen et al., "Haptoglobin is synthesized during granulocyte differentiation, stored in specific granules, and released by neutrophils in response to activation," Blood, vol. 108, no. 1, pp. 353-361, 2006.

[36] L. C. Jacobsen, K. Theilgaard-Mönch, E. I. Christensen, and N. Borregaard, "Arginase 1 is expressed in myelocytes/metamyelocytes and localized in gelatinase granules of human neutrophils," Blood, vol. 109, no. 7, pp. 3084-3087, 2007.

[37] S. Ménard, V. Förster, M. Lotz et al., "Developmental switch of intestinal antimicrobial peptide expression," The Journal of Experimental Medicine, vol. 205, no. 1, pp. 183-193, 2008.

[38] H. Renz, P. Brandtzaeg, and M. Hornef, “The impact of perinatal immune development on mucosal homeostasis and chronic inflammation," Nature Reviews Immunology, vol. 12, no. 1, pp. 9-23, 2012.

[39] C. Atuma, V. Strugala, A. Allen, and L. Holm, "The adherent gastrointestinal mucus gel layer: Thickness and physical state in vivo," The American Journal of Physiology-Gastrointestinal and Liver Physiology, vol. 280, no. 5, pp. G922-G929, 2001.

[40] C. H. F. Hansen, L. S. F. Andersen, L. Krych et al., "Mode of delivery shapes gut colonization pattern and modulates regulatory immunity in mice," Journal of Immunology, vol. 193, no. 3, pp. 1213-1222, 2014.

[41] J. Wu, D. N. Kakoola, N. I. Lenchik, D. M. Desiderio, D. R. Marshall, and I. C. Gerling, "Molecular phenotyping of immune cells from young NOD mice reveals abnormal metabolic pathways in the early induction phase of autoimmune diabetes," PLoS ONE, vol. 7, no. 10, Article ID e46941, 2012.

[42] O. Vaarala, "Leaking gut in type 1 diabetes," Current Opinion in Gastroenterology, vol. 24, no. 6, pp. 701-706, 2008.

[43] C. Alam, E. Bittoun, D. Bhagwat et al., "Effects of a germfree environment on gut immune regulation and diabetes progression in non-obese diabetic (NOD) mice," Diabetologia, vol. 54, no. 6, pp. 1398-1406, 2011. 
[44] P. Pozzilli, A. Signore, A. J. K. Williams, and P. E. Beales, "NOD mouse colonies around the world-recent facts and figures," Immunology Today, vol. 14, no. 5, pp. 193-196, 1993.

[45] A. Khosravi, A. Yáñez, J. G. Price et al., "Gut microbiota promote hematopoiesis to control bacterial infection," Cell Host and Microbe, vol. 15, no. 3, pp. 374-381, 2014.

[46] I. Puga, M. Cols, C. M. Barra et al., "B cell-helper neutrophils stimulate the diversification and production of immunoglobulin in the marginal zone of the spleen," Nature Immunology, vol. 13, no. 2, pp. 170-180, 2012.

[47] F. M. Wolber, E. Leonard, S. Michael, C. M. Orschell-Traycoff, M. C. Yoder, and E. F. Srour, "Roles of spleen and liver in development of the murine hematopoietic system," Experimental Hematology, vol. 30, no. 9, pp. 1010-1019, 2002.

[48] N. B.-V. Paassen, L. M. P. Loonen, J. Witte-Bouma et al., "Mucin Muc2 deficiency and weaning influences the expression of the innate defense genes Reg $3 \beta, \operatorname{Reg} 3 \gamma$ and angiogenin-4," PLoS ONE, vol. 7, no. 6, Article ID e38798, 2012.

[49] A. Bergström, M. B. Kristensen, M. I. Bahl et al., "Nature of bacterial colonization influences transcription of mucin genes in mice during the first week of life," BMC Research Notes, vol. 5, article 402, 2012.

[50] J. L. Sonnenburg, L. T. Angenent, and J. I. Gordon, "Getting a grip on things: how do communities of bacterial symbionts become established in our intestine?" Nature Immunology, vol. 5, no. 6, pp. 569-573, 2004.

[51] K. Vehik and D. Dabelea, "Why are C-section deliveries linked to childhood type 1 diabetes?” Diabetes, vol. 61, no. 1, pp. 36-37, 2012.

[52] F. Hildebrand, A. T. L. Nguyen, B. Brinkman et al., "Inflammation-associated enterotypes, host genotype, cage and interindividual effects drive gut microbiota variation in common laboratory mice," Genome Biology, vol. 14, article R4, 2013. 


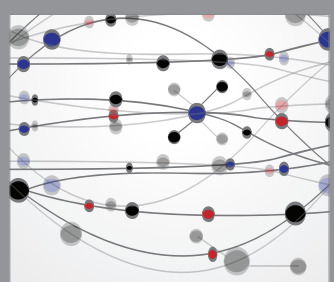

The Scientific World Journal
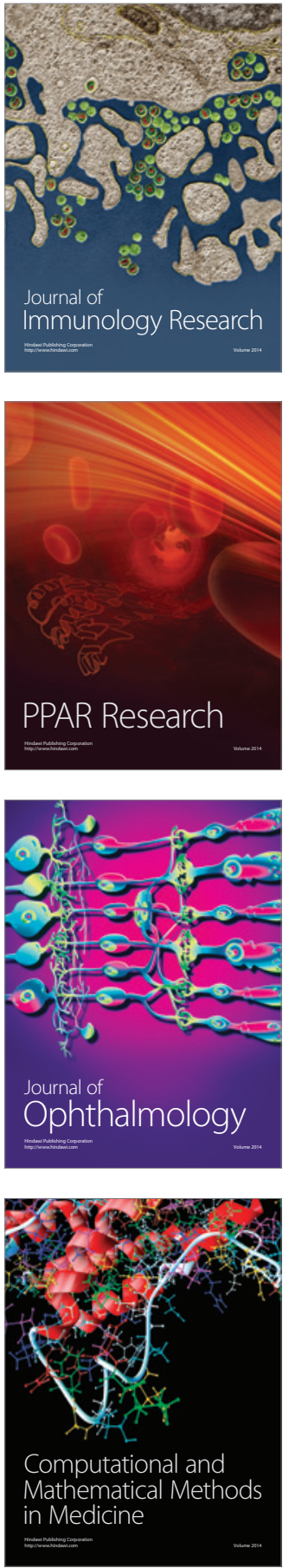

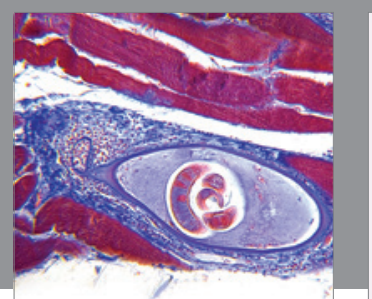

Gastroenterology Research and Practice

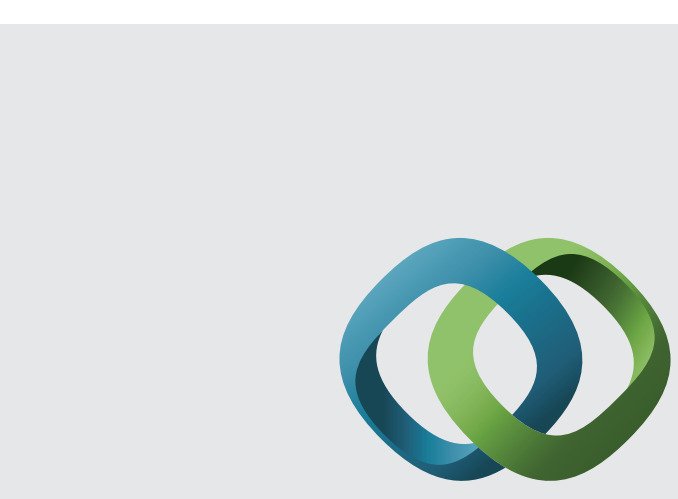

\section{Hindawi}

Submit your manuscripts at

http://www.hindawi.com
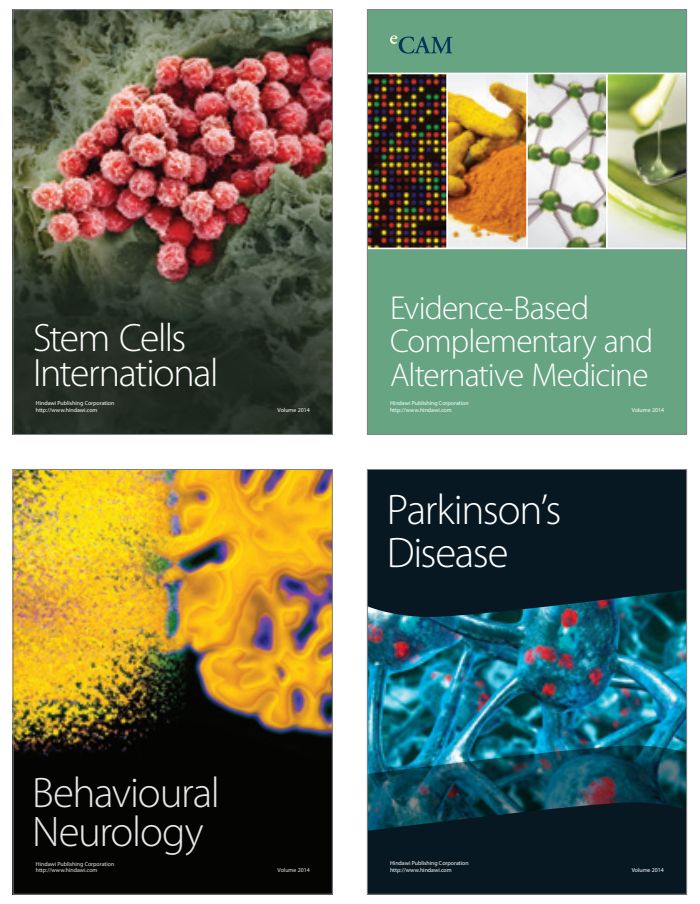
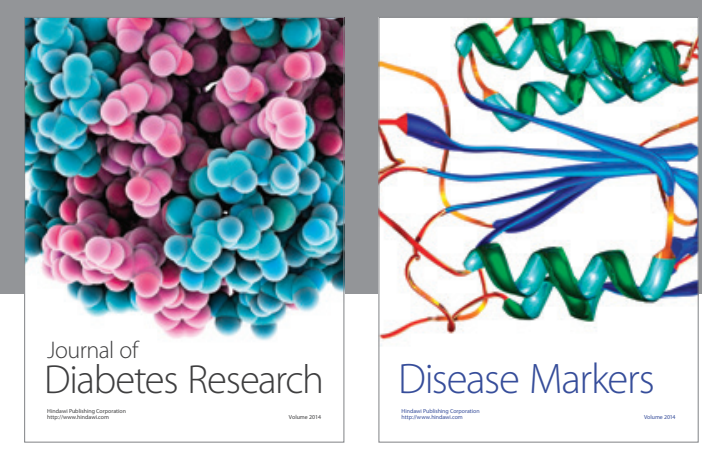

Disease Markers
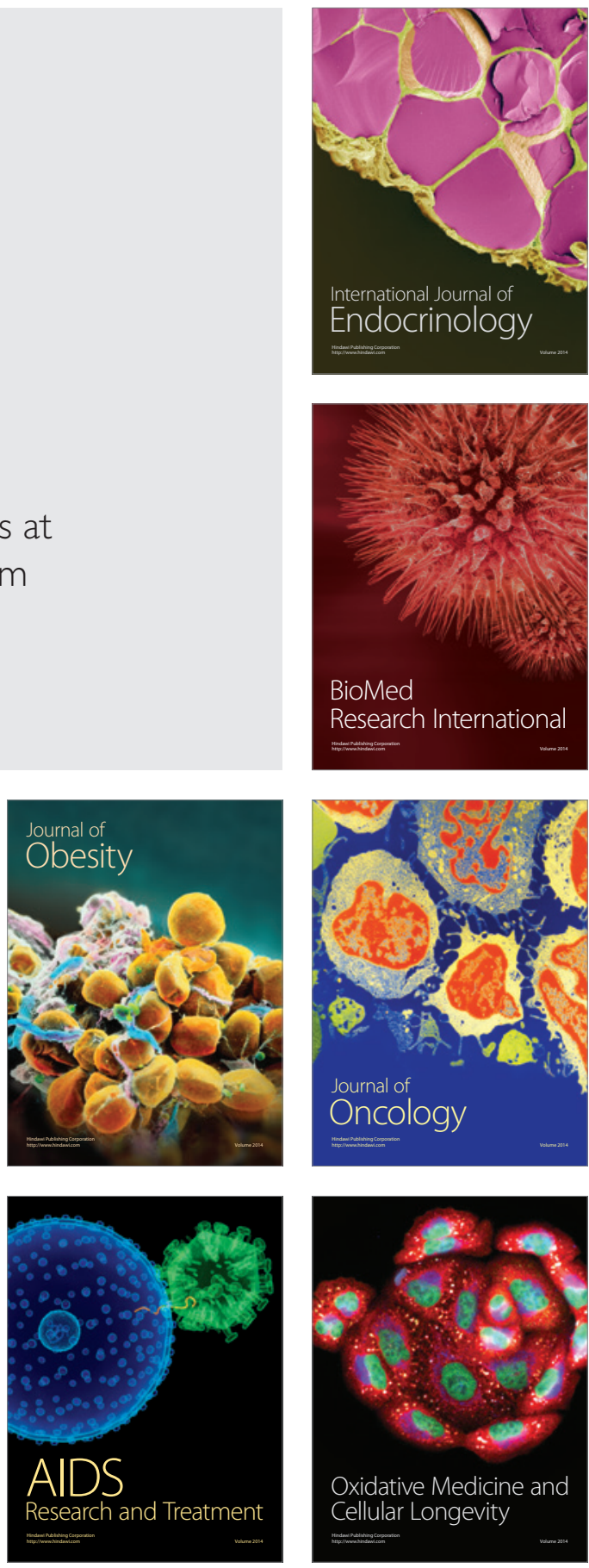